\title{
Modeling the effect of flow-induced mechanical erosion during coffee filtration
}

\author{
Chaojie Mo, ${ }^{1}$ Richard Johnston, ${ }^{2}$ Luciano Navarini, ${ }^{3}$ and Marco Ellero ${ }^{1,4,5}$ \\ 1) Basque Center for Applied Mathematics (BCAM), Alameda de Mazarredo 14, 48400 Bilbao, \\ Spain \\ 2) Faculty of Science and Engineering, Swansea University, Swansea, SA1 8EN, UK \\ 3) Illycaffè S.p.A, Via Flavia 110, Trieste 34147, Italy \\ ${ }^{4)}$ Zienkiewicz Centre for Computational Engineering (ZCCE), Swansea University, Bay Campus, Swansea SA1 8EN, \\ $U K$ \\ 5)IKERBASQUE, Basque Foundation for Science, Calle de María Díaz de Haro 3, 48013 Bilbao, \\ Spain
}

(Dated: 6 September 2021)

\begin{abstract}
The espresso extraction process involves a complex transport inside a geometry-changing porous medium. Large solid grains forming the majority of the porous medium can migrate, swell, consolidate and they can also morphologically change during flow, i.e. being mechanically eroded by hydrodynamic forces. These processes can, in turn, have a significant back-effect on the flow and the related coffee extraction profiles. In this article, we devise a bottom-up erosion model in the framework of smoothed dissipative particle dynamics to consider flow-induced morphological changes of the coffee grains. We assume that the coffee grains are not completely wetted and remain brittle. We found that heterogeneity in both the filtration direction and the transverse direction can be induced. The former is controlled by the angle of internal friction while the latter is controlled by both the cohesion parameter and the angle of internal friction. Not restricted to the modeling of espresso extraction, our model can also be applied to other eroding porous media. Our results suggest that, under ideal porous flow conditions, we can control the heterogeneity (in both the pressure drop direction and the transverse direction) of an eroding medium by tuning the yield characteristics of the eroding material.
\end{abstract}

\section{INTRODUCTION}

Coffee is one of the most popular beverages in the world. Its aroma, taste and mouthfeel is very sensitive to the brewing method and the control of many brewing parameters. Among many methods for preparing coffee (filter drip, pods and capsules, Turkish coffee, French press coffee), espresso coffee is brewed around the world, and forms part of social and cultural activity due to its uniquely rich taste, smell and mouthfeel. Espresso is prepared by percolating hot water $\left(\approx 92^{\circ} \mathrm{C}\right)$ under very high pressure $(\approx 9$ bar $)$ through a tamped cake of ground roasted coffee in $20 \sim 30$ seconds $1 \mid 2$. Despite its popularity, espresso coffee extraction is still poorly understood due to its complexity, but a quantitative understanding of its dynamics is a crucial step to systematically improve the cup quality 3 . Espresso extraction involves flows through a porous medium undergoing geometric changes ${ }^{4}$, solubilization of many hydrophilic substances 5 , emulsification of insoluble coffee oils ${ }^{6}$, suspension of solid coffee cell-wall fragments (fines) ${ }^{6}$, and $\mathrm{CO}_{2}$ degassing and supersaturation ${ }^{7}$. Each of the aforementioned physical process is itself intrinsically complex and, more importantly, they are interconnected. Therefore, the investigation of the espresso extraction is a difficult challenge. For example, the solubilization and emulsification processes will change the viscosity of the liquid, affecting the flow through the porous coffee cake, and the change of the flow will in return affect the solubilization and emulsification. For a detailed description of the complex physical processes occurring during coffee extraction the reader is referred to the complete review by Melrose et a ${ }^{899}$. Physics of coffee extraction has been modelled in some earlier studies $10[11$ and some very recent works 12,15 . Most of these studies rely on continuum models for the concentrations fields suitably coupled to hydrodynamic effects. Models to simulate the espresso extraction taking into account the detailed physical processes at the mesoscopic levels are rare: numerical models have been devised to consider the solubilization of extraction of soluble substances $16 / 17$, the migration of fines 16 , the erosion and swelling of the coffee cake ${ }^{18}$. In this article, we focus on the effect of "mechanical" erosion, the process that the flow through the porous medium removes the solid material by mechanically fracturing surfaces exposed to the liquid and therefore keeps altering the boundaries. This will be different from a similar process of mass removal which is dissolution, related to boundary remodelling by surface solubilization which could be classified as "chemical" erosion and will not be considered here.

The erosion process is prevalent in nature, helping to form some distinguishable morphological patterns 1920]. During an erosion process, the solid boundary is shaped by the flow, and the change of the boundary will in return influence the flow. This feedback mechanism is responsible for many patterns formed in an eroding porous medium $21+23$ and it has been the subject of many theoretical works. In the work of Mahadevan et $a l^{22}$ an effective continuum multiphase model was devised, which is able to reproduce channelization in porous media in qualitative agreement with experiments. A very similar model excluding the deposition effects was used by Derr et $a t^{21}$ to study the branching in a frangible porous medium. Kudrolli and Clote ${ }^{24}$ also used an effective model to simulate the channelization in eroding porous medium. These continuum effective models use the Darcy's law to model the flow, 
whereas the erosion is considered to depend on local pressure gradient $^{2122]}$ or local flow velocity 24 . In the work of Jäger et $a i^{25}$, the Navier-Stokes equations were solved using the lattice Boltzmann method (LBM), and erosion was assumed to depend on the local shear stress on the solid surfaces. It was found that constant pressure drop results in either completely clogged configuration or completely eroded configuration, while a constant flux will result in at least one stable channel. By including hydraulic pressure gradient as another factor that can induce erosion, Jäger et a ${ }^{26}$ were able to recover erosive bursts in porous medium. The power law distribution of pressure loss jumps obtained from their simulation was in excellent agreement with experiment. Chiu et $a l^{27}$ resolved the Stokes equation with the boundary integral method and also consider the erosion to be induced by shear stress. The porosity-tortuosity relationship, the asymptotic dispersion rate and the pore size distribution during the erosion process were studied. Matias et a 18 used the LBM and shear-erosion model to study the erosion and swelling of coffee cake. They found that when the flux is constant the final state depends only on the erosion dynamics, but when the pressure drop is constant the final state depends on the competition between the erosion and swelling.

When modeling eroding porous media, the common practice is to assume that the erosion rate is proportional to the difference between local shear stress and a threshold. This "shear-erosion model" is supported by erosion experiments on bodies molded from clay 28/29. Despite this, in geoscience there are also studies 30 that not only consider the erosion induced by shear stress but also take the yield of the solid material into account. Clearly, different materials have different erosion dynamics. It is not known whether the shear-erosion model is suitable for the porous coffee cake studied here. After grind, the roasted coffee beans break into small grains of a bimodal size distribution 33 . The first peak of the distribution function is always at around $30 \sim 40 \mu \mathrm{m}$, representing the so-called "fines", i.e. inner walls cellular fragments. The exact position of the second peak depends on the grinder, but normally it is at $200 \mu \mathrm{m} \sim 1000 \mu \mathrm{m}$. It should be noted that the ground coffee grains are not entirely solid, but full of pores. These pores are either large pores $(30 \mu \mathrm{m} \sim 60 \mu \mathrm{m})$ that are cell-pockets or small pores of nanoscale on the cellwalls 33 . The residual $\mathrm{CO}_{2}$ generated during the roasting process are trapped in these pores, and the degassing and supersaturation of this $\mathrm{CO}_{2}$ is partially responsible for the formation of crema that make the mouthfeel of espresso unique $e^{7 / 34}$. To date, no experimental data is available to show the extend this kind of material is eroded by the fluid during filtration. We know that roasting renders coffee beans more brittle ${ }^{35}$. During the extraction process the coffee grains are wetted again and probably become more ductile but, according to some early studies $4 \sqrt[36]{ }$, the transient phase in which wetting and percolation occur simultaneously last about 5 seconds. So even though the coffee grains are more ductile after wetting, this is a relatively slow process. Besides, scanning electron microscopy observation shows that immersed coffee grain has gas entrapped, indicating that it is not fully wetted 37 . The coffee grains probably remain sufficiently brit- tle in the early stage of extraction. This feature of coffee grains inspires us to use an erosion model that incorporate the Mohr-Coulomb failure criterion, which is more suitable to describe the failure of brittle materials. Therefore, in this article, we propose a bottom-up mesoscopic erosion model developed in the framework of smoothed dissipative particle dynamics (SDPD). This model can be seen as a combination of the Mohr-Coulomb yield criterion ${ }^{38[39}$ and shear-erosion. Therefore, it is a pressure-dependent erosion model. Although the Mohr-Coulomb criterion and similar criterion (e.g. DruckerPrager criterion) have been incorporated into erosion model in simulations of sediment erosion 30 , the outcome of applying the Mohr-Coulomb criterion to the erosion of porous medium has not been studied. With our erosion model, we will investigate the effects of the Mohr-Coulomb parameters on the erosion characteristics of a porous medium.

This paper is organized as follows. In section III the simulation models are introduced, including the SDPD model (section II A), the erosion model (section II B and the porous medium model (section IID). The erosion model is also validated in section $\amalg \mathrm{C}$ and discussed in section $\amalg \mathrm{E}$. In section III the time-dependent eroding geometry and characteristics are first presented (section III A), then we discuss the effects of the cohesion parameter in section III B and of the angle of internal friction in section IIIC. Finally, conclusions are reported in section IV

\section{COMPUTATIONAL MODEL}

\section{A. Smoothed dissipative particle dynamics}

In our model the flow is governed by the Navier-Stokes equations. To solve the Navier-Stokes, we employ the smoothed dissipative particle dynamics (SDPD) method 40 , which is a particle-based mesoscopic hydrodynamics approach. SDPD is derived through a Lagrangian discretization of the Navier-Stokes equations similar to the smoothed particle hydrodynamics (SPH) method ${ }^{43}$, with the inclusion of thermal fluctuations following the dissipative particle dynamics (DPD) approach 44 , albeit with the proper thermodynamics scaling 46 . Thus it can be seen as a generalization of SPH for fluctuating hydrodynamics. We employ a SDPD version which conserves angular momentum 41 , as it can be crucial for some problems ${ }^{47 / 48}$. In SDPD, each particle can be considered as a small fluid volume (or Lagrangian discretization point) characterized by a position $\boldsymbol{r}_{i}$, velocity $\boldsymbol{v}_{i}$, and mass $m_{i}$. In addition, each SDPD particle possesses a spin angular velocity $\psi_{i}$ and moment of inertia $I_{i}$ introduced for the enforcement of angular momentum conservation 41 .

SDPD particles $i$ and $j$ interact through four pairwise forces, including conservative $\boldsymbol{F}_{i j}^{C}$, dissipative $\boldsymbol{F}_{i j}^{D}$, rotational 
$\boldsymbol{F}_{i j}^{R}$ and stochastic $\tilde{\boldsymbol{F}}_{i j}$ forces given by

$$
\begin{gathered}
\boldsymbol{F}_{i j}^{C}=\left(\frac{P_{i}}{d_{i}^{2}}+\frac{P_{j}}{d_{j}^{2}}\right) F_{i j} \boldsymbol{r}_{i j}, \\
\boldsymbol{F}_{i j}^{D}=-\gamma_{i j}\left[\boldsymbol{v}_{i j}+\left(\boldsymbol{e}_{i j} \cdot \boldsymbol{v}_{i j}\right) \boldsymbol{e}_{i j}\right], \\
\boldsymbol{F}_{i j}^{R}=-\gamma_{i j} \frac{\boldsymbol{r}_{i j}}{2} \times\left(\boldsymbol{\psi}_{i}+\boldsymbol{\psi}_{j}\right), \\
\tilde{\boldsymbol{F}}_{i j}=\sigma_{i j}\left(d \overline{\mathscr{W}}_{i j}^{s}+\frac{1}{3} \operatorname{tr}\left[d \mathscr{W}_{i j}\right] \mathbf{1}\right) \cdot \frac{\boldsymbol{e}_{i j}}{d t},
\end{gathered}
$$

where $\boldsymbol{r}_{i j}=\boldsymbol{r}_{i}-\boldsymbol{r}_{j}, \boldsymbol{v}_{i j}=\boldsymbol{v}_{i}-\boldsymbol{v}_{j}$, and $\boldsymbol{e}_{i j}=\boldsymbol{r}_{i j} / r_{i j}$. Particle number density $d_{i}$ is computed as $d_{i}=\sum_{j} W_{i j}$ using a smoothing kernel function $W_{i j}=W\left(r_{i j}\right)$ that vanishes beyond a cutoff radius $r_{c}$ and defines a non-negative function $F_{i j}$ through the equation $\nabla_{i} W_{i j}=-\boldsymbol{r}_{i j} F_{i j}$. Then, particle mass density is given by $\rho_{i}=m_{i} d_{i}$. The pressure $P_{i}$ is determined by the equation of state (EoS) $P_{i}=P_{0}\left(d_{i} / d_{0}\right)^{v}-P_{\mathrm{b}}$, where $d_{0}$ is the average number density, $P_{0}$ and $v$ are parameters controlling the sound speed $c_{s}=\sqrt{P_{0} v / d_{0}}$, and $P_{\mathrm{b}}$ relates to the background pressure. Furthermore, $\gamma_{i j}$ and $\sigma_{i j}$ are the corresponding force amplitudes

$$
\gamma_{i j}=\frac{20 \eta}{7} \frac{F_{i j}}{d_{i} d_{j}}, \quad \sigma_{i j}=2 \sqrt{k_{\mathrm{B}} T \gamma_{i j}}
$$

where $\eta$ is the fluid dynamic viscosity, $T$ is the equilibrium temperature, and $k_{\mathrm{B}}$ is the Boltzmann constant. Eq. (1) also contains a matrix of independent Wiener increments $d \mathscr{W}_{i j}$ with its trace $\operatorname{tr}\left[d \mathscr{W}_{i j}\right]$ and the traceless symmetric part $d \overline{\mathscr{W}}_{i j}^{s}=\frac{1}{2}\left(d \mathscr{W}_{i j}+d \mathscr{W}_{j i}\right)-\frac{1}{3} \operatorname{tr}\left[d \mathscr{W}_{i j}\right]$, and the time step $d t$.

The evolution of particle positions, translational and angular velocities is obtained by integration of the following equations of motion

$$
\begin{gathered}
\dot{\boldsymbol{r}}_{i}=\boldsymbol{v}_{i}, \\
m_{i} \dot{\boldsymbol{v}}_{i}=\sum_{j} \boldsymbol{F}_{i j}=\sum_{j}\left(\boldsymbol{F}_{i j}^{C}+\boldsymbol{F}_{i j}^{D}+\boldsymbol{F}_{i j}^{R}+\tilde{\boldsymbol{F}}_{i j}\right), \\
\dot{\psi}_{i}=\frac{1}{2 I_{i}} \sum_{j} \boldsymbol{r}_{i j} \times \boldsymbol{F}_{i j},
\end{gathered}
$$

using the velocity-Verlet algorithm 49 .

In this work, we only perform two dimensional (2D) simulations, the smoothing kernel is represented by the $2 \mathrm{D}$ Lucy function $\underline{50}$

$$
W(r)=\frac{5}{\pi r_{c}^{2}}\left(1+3 \frac{r}{r_{c}}\right)\left(1-\frac{r}{r_{c}}\right)^{3},
$$

where $r$ is the shorthand for $\left\|\mathbf{r}_{i j}\right\|$, and $r_{c}$ is the cutoff radius.

\section{B. Erosion model}

We model the solid coffee grain using a cluster of SDPD particles. Harmonic springs are used to interconnect these particles with their nearest and second nearest neighbors. We consider a material that is brittle. The basic principle for the erosion is that, if a material particle on the surface of a coffee grain is experiencing a force exerted by the fluid, and this force is larger than a given threshold, and is in a direction leaving the bulk of the grain, then this particle is eligible to be eroded. The probability of the erosion depends on the force on the particle.

The implementation of the erosion model in SDPD is as follows:

(i) All the grain particles are connected by harmonic bonds/springs (see Fig. 1).

(ii) A bond number criterion is set as $N_{b}$, if the number of bonds connected to a particle is smaller than this criterion, that particle is labeled as surface particle, otherwise as internal particles. In two dimensions $N_{b}=8$ if the grain particles are in sq2 lattice (2d lattice that defines a square unit cell with one basis atom at the corner and one at the center of the square), but if we want those particles at concave surface are also labeled as internal particles $N_{b}$ can be set smaller. Here we use $N_{b}=7$.

(iii) Internal particles are fixed. The surface particles can move freely (constrained by the bonds).

(iv) Every $N_{\text {freq }}$ time steps all the bonds are checked. A bond is eligible to break if the following two conditions are satisfied:

- The bond is stretched by a distance longer than a length threshold $l_{c}^{i}=l_{0}^{i}+\Delta l_{c}$, here $l_{0}^{i}$ is the equilibrium length of the bond, $\Delta l_{c}$ is a global stretch threshold;

- Among all the bonds connecting a surface particle this bond is the one with the largest stretch.

The probability for that bond to break is $p_{i}=p_{0}\left(l_{i}-\right.$ $\left.l_{c}^{i}\right)=p_{0}\left(\Delta l_{i}-\Delta l_{c}\right) \leq 1$, here $l_{i}$ is the length of the bond, $\Delta l_{i}=l_{i}-l_{0}^{i}$.

(v) If all the bonds connecting a grain particle are broken, this particle will move freely along with the flow and become indistinguishable with the fluid particles. Therefore deposition is not considered in this work.

(vi) With bonds connecting the surface particles broken, some internal particles will be exposed to the outside liquid domain and become new surface particles. The erosion process therefore continues on the newly exposed surface.

The erosion phenomenon naturally arises following these rules.

Let's look at the microscopic picture of the erosion. As shown in Fig. 1] we pick one surface particle for analysis. In the magnified inlet, the surface particle is under an external force $\mathbf{F}$ exerted by the fluid. This force is mainly balanced by springs connecting this surface particle and the interior particles. We use $l_{i}$ to denote these springs and their lengths. The vector $\mathbf{l}_{i}$ points from an interior particle towards the surface particle and $\hat{\mathbf{l}}_{i}$ is its corresponding unit vector. Assuming the 


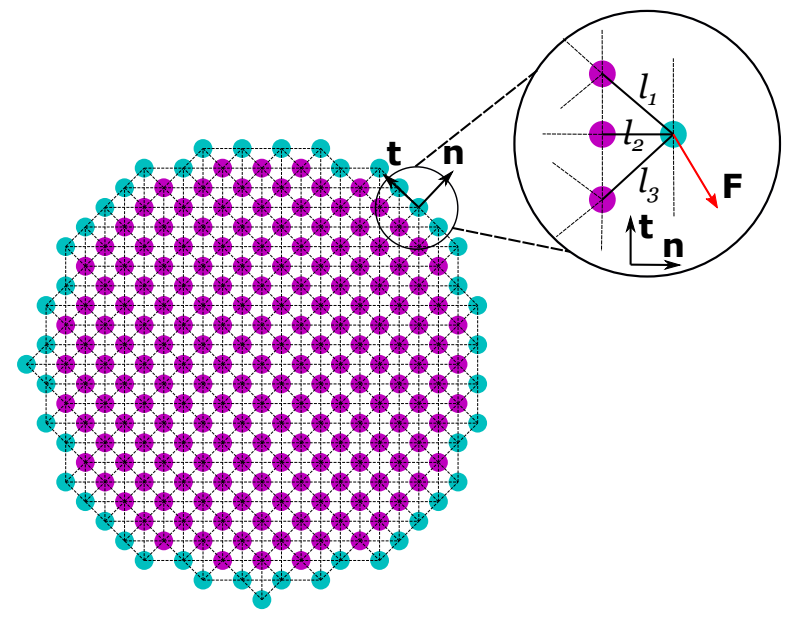

FIG. 1. Schematic of the erosion model. Purple particles: internal particle; cyan particles: surface particles. In the figure, $\mathbf{t}$ is the tangential unit vector, $\mathbf{n}$ is the normal unit vector.

springs coefficient $k$ is very large and the displacement $\mathfrak{d}$ of the surface particle is small compared to the spring length, the force balance indicates that

$$
\mathfrak{d}=\left(\sum_{i} \hat{\mathbf{l}}_{i} \hat{\mathbf{l}}_{i}\right)^{-1} \cdot \frac{\mathbf{F}}{k} .
$$

Then the stretch of each spring is

$$
\Delta l_{i}=\hat{\mathbf{l}}_{i} \cdot \mathfrak{d} .
$$

According to the erosion rules a spring is eligible to break with a probability

$$
p_{i}=\min \left\{p_{0}\left(\Delta l_{i}-\Delta l_{c}\right), 1\right\},
$$

when $\Delta l_{i}-\Delta l_{c}>0$ and when this spring has the largest stretch among all the springs connecting the surface particle. So the springs $l_{i}$ will break in sequence, with each break changing the tensor $\sum_{i} \hat{\mathbf{l}}_{i} \hat{\mathbf{l}}_{i}$ in a non-continuous way. Given the $p_{i}$ the expected living time (measured from the instant that the spring becomes eligible to break) for a spring is

$$
T_{i}=N_{\text {freq }} \Delta t / p_{i},
$$

where $N_{\text {freq }} \Delta t$ is time interval to check all the springs and perform the break action. The mass erosion rate is therefore

$$
k_{\mathrm{er}}=\frac{m}{\sum_{i \in S_{b}} T_{i}},
$$

where $m$ is the mass of the particles, $S_{b}$ is the break sequence. The break sequence is only known during computation because it is difficult to determined which spring has the largest stretch beforehand and each break of spring induces an abrupt change on the tensor $\sum_{i} \hat{\mathbf{l}}_{i} \hat{\mathbf{l}}_{i}$. Note that $T_{i}$ must be calculated following the break sequence. Finally, the last spring connecting the surface particle can be excluded from the break sequence since one spring cannot fully restrict the movement

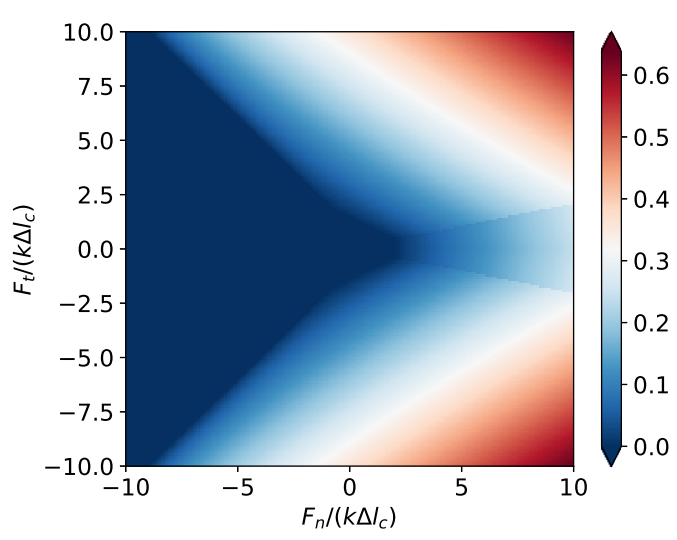

FIG. 2. Erosion rates at different external forces.

of the surface particle and our model relabel the interior particle to be the new surface particle in such a situation. Given external force $\mathbf{F}$ and solving Eq. (5) - Eq. (9) we can get the mass erosion rate.

The above analysis is applicable to particle configuration not restricted to that shown in Fig. 1. Given the direction vectors of the connecting springs between the surface particle and interior particle the erosion rate can be determined. The particles are in sq2 lattice as shown in Fig. 1, so we have

$$
\begin{gathered}
\hat{\mathbf{l}}_{1}=\left[\frac{\sqrt{2}}{2},-\frac{\sqrt{2}}{2}\right]^{\mathrm{T}}, \\
\hat{\mathbf{l}}_{2}=[1,0]^{\mathrm{T}}, \\
\hat{\mathbf{l}}_{3}=\left[\frac{\sqrt{2}}{2}, \frac{\sqrt{2}}{2}\right]^{\mathrm{T}} .
\end{gathered}
$$

Prescribing $m=1, N_{\text {freq }} \Delta t=0.01, k=100, \Delta l_{c}=0.001$, $p_{0}=1.0$, the contour of erosion rate at different external force $\mathbf{F}=F_{t} \mathbf{t}+F_{n} \mathbf{n}$ can be obtained using the above equations. The result is shown in Fig. 2 It demonstrates a unique erosion model compared with those in literatures $18 / 21|22| 2426151 / 52$. In literatures, some erosion models assume that the erosion rate is proportional to the difference between the shear stress and the threshold stres\$1825-27; other erosion models assume that the erosion is dependent on the local flow velocity 2453; in effective models using Darcy's law it is usually assumed that the erosion rate depends on the difference between the local pressure gradient and a threshold 12122 . In the model presented in this work, negative normal stress impedes the erosion while positive stress enhances it. Figure 3 (a) specifically shows how the erosion rate depends on the tangential force when the normal force is kept constant. It shows a linear relationship between the tangential force and the erosion rate in the regime where the tangential force is larger than a given threshold. Figure 3 (b) shows that the tangential force threshold is linear against the normal force when the normal force is pointing inward. These indicate that the present model is actually a combination of the shear-erosion model and the Mohr-Coulomb yield criterion ${ }^{39}$, and it is therefore pressure-dependent. There are three relevant controlling parameters present in this model: 
(a)

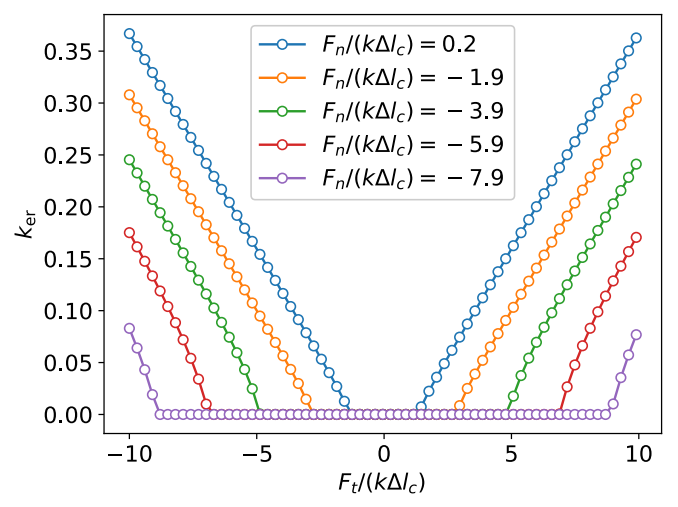

(b)

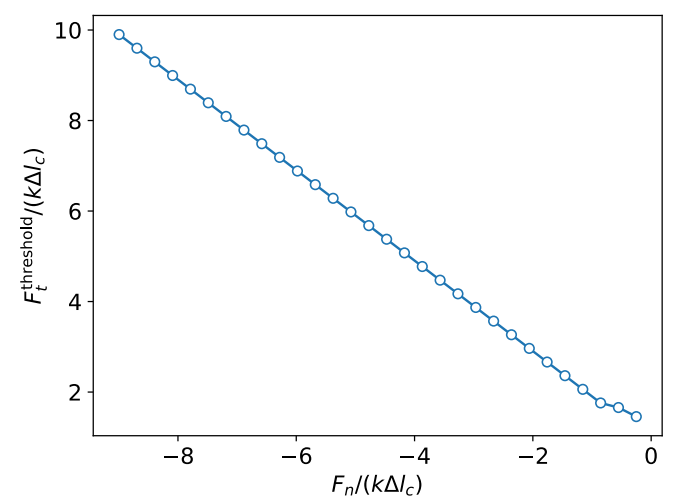

FIG. 3. (a) Erosion rates as a function of tangential force at different normal forces. (b) Tangential force threshold at different normal forces.

the shear erosion coefficient $C_{\mathrm{E}}$, the cohesion parameter $c$ and the angle of internal friction $\psi$. The erosion rate can be expressed as

$$
\begin{gathered}
k_{\mathrm{er}}=\max \left\{C_{\mathrm{E}}\left(\tau-\tau_{c}\right), 0\right\} \\
\tau_{c}=\sigma \tan \psi+c,
\end{gathered}
$$

where $\tau_{c}$ is the shear erosion threshold, $\sigma$ is the applied normal stress. The shear erosion constant is

$$
C_{\mathrm{E}} \sim C_{\mathrm{E}}^{\prime} \equiv \frac{m p_{0}(\Delta x)^{d-1}}{k N_{\text {freq }} \Delta t},
$$

where $\Delta x$ is the average particle distance, $d$ is the dimension of the problem. In Eq. (12), $C_{\mathrm{E}}$ is the shear erosion constant, but its analytic expression cannot be obtained, so we estimate its value using $C_{\mathrm{E}}^{\prime}, C_{\mathrm{E}}^{\prime}$ is obtained by analysing a particle constrained only by a bond parallel to the tangential direction of the surface and is being eroded by shear flow. On these conditions the expression of $C_{\mathrm{E}}^{\prime}$ is obtained using Hooke's law and Eq. (7)-Eq. (9). Note that we do not seek to get an accurate value of $C_{\mathrm{E}}$, we only need $C_{\mathrm{E}}^{\prime}$ to estimate the typical erosion time scale of a particle. The cohesion parameter $c$ is controlled by $\Delta l_{c}$ as will be discussed later. We will also devise a simple scheme to control the angle of internal friction $\psi$ later.

Returning to the coffee cake we are studying, we know from the literature ${ }^{633}$ that there is a significant amount of microand nano- pores inside the solid grains. $\mathrm{CO}_{2}$ generated during the roasting process occupy these micro-pores, so the fluid can not penetrate into all the voids inside grains at an instant 7 $^{34}$ [37. The microscopic detail of how those gases are driven out of the grains and dissolve into the water - which in turn generate the foam and espresso crema - is still unclear. The process might involve the compacting, swelling and erosion of the coffee ground under pressure. In any case, the existence of gas in the micro- and nano- pores allows temporary unbalances of pressures at the liquid-solid interfaces and justifies the use of a pressure-dependent erosion model in this system. There are two mechanics through which the existence of entrapped gas could leads to pressure-dependent erosion: 1) the solid grains are prone to be consolidated due the pressure unbalance, re- sulting in internal friction; 2) Grains not fully wetted will remain sufficiently brittle, and the failure of brittle material is usually pressure dependent. On the other hand, if we were studying the more classic erosion of sand or soil, it would be obvious that only the pressure gradient and the shear stress are important since the water can penetrate into all the voids in the relevant timescale of the process.

\section{Model validation}

The erosion model is validated using a simple shear erosion simulation as depicted in Fig. 4 (a). In this configuration, a shear flow is generated by the moving upper wall. The grains are then eroded by the shear flow. With the erosion of the grains progressing, the height of the fluid region $L$ will increase. The corresponding SDPD model is shown in Fig. 4 (b). The light green and yellow particles represent solid walls. The lower wall is fixed and the upper wall moves horizontally at a constant speed $v_{x}$. The dark green particles represent the fluid, and the dark purple particles represent erodible grains modelled using our erosion model (Fig. 1). Boundaries are periodic in the $x$ direction. We set $\Delta l_{c}=0$ leading to a zero erosion threshold. Then the evolution of $L$ can be described by a simple equation:

$$
\frac{\mathrm{d} L}{\mathrm{~d} t}=\frac{\eta v_{x} k_{w}}{L}
$$

where $\eta$ is the fluid viscosity, $k_{w}$ is the wall erosion rate. The solution is

$$
L=\sqrt{2 k_{w} v_{x} \eta t+L_{0}^{2}} .
$$

where $L_{0}$ is the value of $L$ at $t=0$. There is a simple relationship between the the wall erosion rate $k_{w}$ and the mass erosion rate $k_{\mathrm{er}}$ :

$$
k_{w}=\Delta x k_{\mathrm{er}} / m
$$

where $\Delta x$ is the average particle distance. Moreover, in shear flow the hydrodynamic force on a surface particle is $F_{t}=$ 
(a)

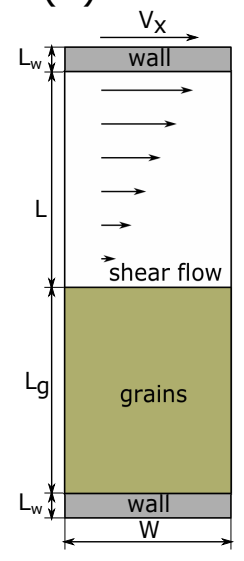

(b)

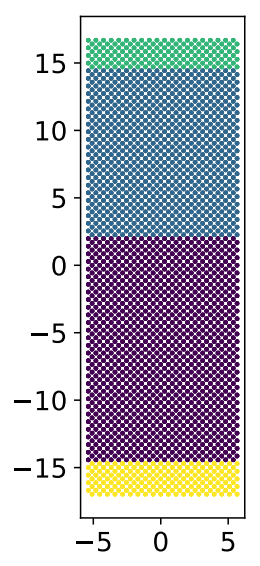

(c)

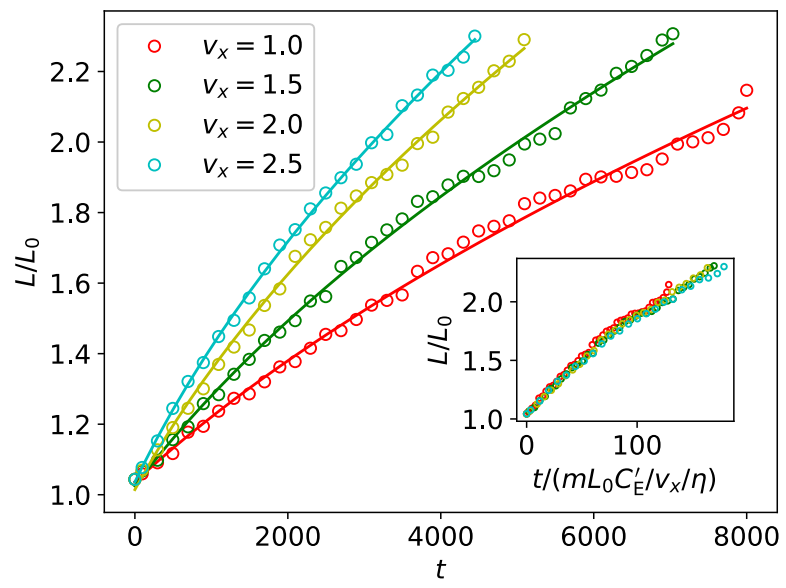

FIG. 4. (a) Schematic of the shear erosion simulation. (b) SDPD model for the shear erosion simulation. $W=11.31, L(t=0)=L_{0}=12.45$, $L_{g}(t=0)=16.8, L_{w}=2.24$. (c) Evolutions of the height of fluid region under shear erosion. The lines are fits using Eq. 14 . The inset shows the same data points but with the time normalized by typical erosion time $t_{\text {ref }}=m L_{0} / C_{E}^{\prime} / V_{x} / \eta$.

$v_{x} \Delta x \eta / L, F_{n}=0$, therefore $k_{w}$ can be determined theoretically. Prescribing $\Delta x=0.4, k=2000, \Delta l_{c}=0, N_{\text {freq }} \Delta t=0.02$, $p_{0}=0.2$, and using the unit vectors $\hat{\mathbf{I}}_{i}$ given in Eq. 10 , we first determine the mass erosion rate using Eq. (5) Eq. (9) then we can determine $k_{w}$ using Eq. (15): the result is $k_{w}=3.3 \times 10^{-4}$. We supplement the parameters $r_{c}=1.6$, $\eta=100, P_{0}=25600, P_{0}-P_{b}=80, v=7, d_{0}=6.25, m=1$, $k_{\mathrm{B}} T=1 \mathrm{e}-6$ for the SDPD model and perform simulations with $v_{x}$ varying from 1.0 to 2.5 . From simulation results the $k_{w}$ can be obtained by fitting the evolution of $L$ using Eq. (14). In Fig. 4 (c), the evolutions of $L$ at different $v_{x}$ are presented and they can be well fitted using Eq. (14). The fits suggest that $k_{w}=3.1 \times 10^{-4} \pm 0.1 \times 10^{-4}$ in good agreement with the theoretical prediction. This validates our erosion model for the following analysis on the erosion in a complex porous medium.

\section{Porous medium model}

We build a SDPD porous medium model as shown in Fig. 5 The size of the simulation box is $L_{x} \times L_{y}$. Inside the box, a sample region $\Omega_{s}$ of height $L_{s}$ is filled with 225 solid disks of diameter $D_{0}$. The center of the disks are placed randomly. The solid disks are modelled using the erosion rules presented above. All boundaries are periodic, and at the top of the simulation box the velocity of the fluid is prescribed/reset as $[0,-q]$, therefore the flux is prescribed in our simulations. The situation that the pressure drop is fixed will not be discussed, since in that case the porous medium will be either completely eroded or not eroded at all ${ }^{25}$. In all simulations the diameter $D_{0}$, the fluid density $\rho$ and the viscosity $\eta$ are fixed: $D_{0}=8$, $\rho=6.25, \eta=100$. The superficial rate $q$ is also fixed as 0.08 , leading to a Reynolds number $\operatorname{Re}=D_{0} \rho q / \eta=0.04$. Simulation parameters are summarized in Tab. II if a parameter is not specified explicitly, the value in the table will be used.
The choice of the parameters in Tab. I are justified as follows. Only some of the parameters that has independent dimensions like $\eta, \rho, D_{0}$ are, to some extent, arbitrary, because they can either be selected as governing parameter to scale all other parameters, or their effects on the result is negligible due to low Reynolds condition. The values of the cutoff radius $r_{c}$ and number density $d_{0}$ are selected to ensure that there are enough neighboring particles for each particle. The value of the flow rate $q$ is selected according to the small Reynolds number. $L_{x}$ and $L_{s}$ are sample size, if each grain in our simulation represents a grain of size $200 \mu \mathrm{m}$ the sample is $4.2 \mathrm{~mm} \times 3.5 \mathrm{~mm}$. It is smaller than a realistic coffee cake used for espresso extraction, but it is enough to demonstrate the filtration characteristics we want to show. The total number of the grains $N_{s}$ is selected to match the initial porosity. $P_{0}, P_{b}, v$ are parameters controlling the equation of state, their values are selected to ensure the compressibility of the fluid is very small (the sound speed $\left.c_{s}=\sqrt{P_{0} v / d_{0}}\right)$. And since the effect of the thermal fluctuations is negligible, $k_{\mathrm{B}} T$ is set to be very small. The criterion $N_{b}$ is fixed as 7 for reason explained in section II B. The parameters $k, p_{0}, N_{\text {freq }}$ are parameters controlling the erosion rate, to some extent they are also arbitrary (excerpt that $k$ should be large enough to ensure that the displacement of an eroding surface particle is small), that is why in our presentation of the results, we have use the characteristic erosion time $t_{\mathrm{er}}$, which is calculated using these parameters (see Eq. (12) and discussion in section IIE), to non-dimensionalize our results. We locate the SDPD particles of the grains in a sq2 lattice configuration as shown in Fig. 1, therefore the direction vectors are given by Eq. 10 .

During simulation, the positions and velocity of all the SDPD particles are recorded for post-processing. After the simulation, the evolving porosity (either local or global) can be determined by counting the number of non-fixed particles in the region of interest and divide it by the total number of particles in that region at the specific time. The pore 
(a)

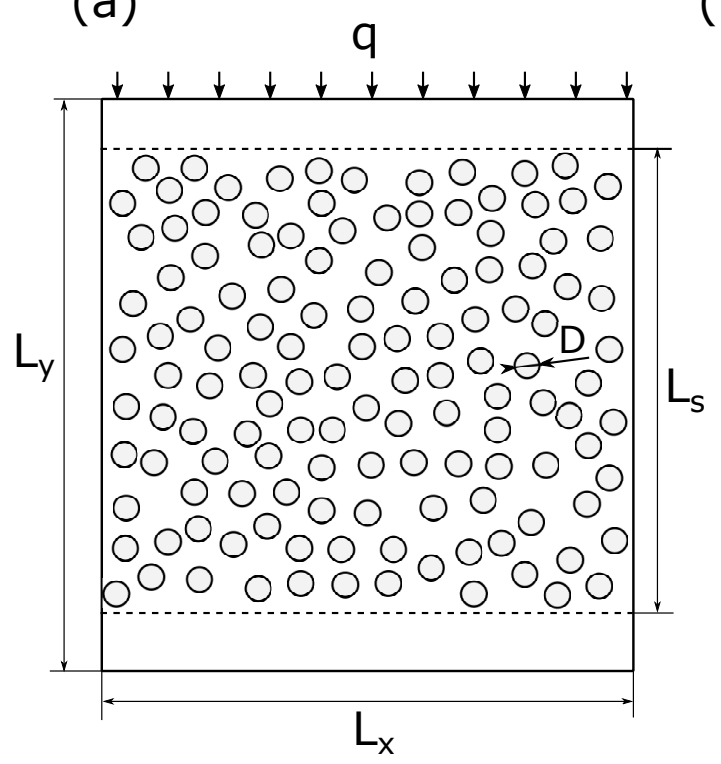

(b)

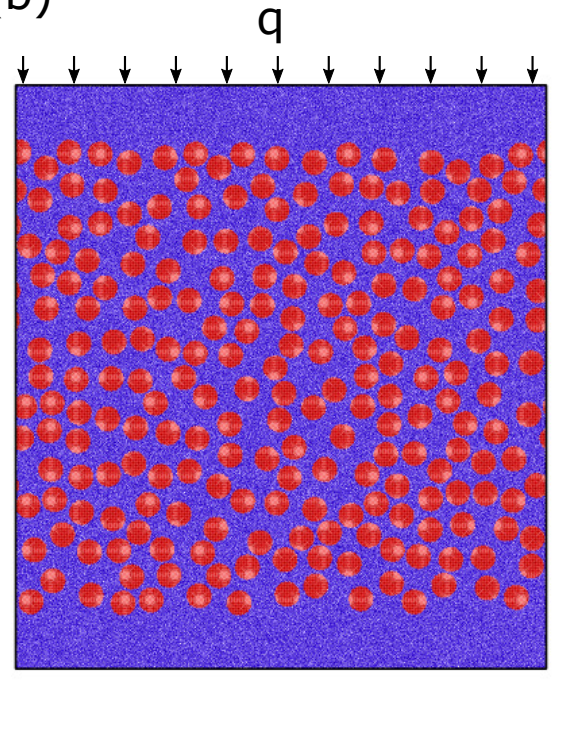

FIG. 5. (a) Schematic of the porous medium model. (b) SDPD model of the porous medium.

TABLE I. Basic parameters used in simulations.

\begin{tabular}{ll}
\hline Basic parameters & Values \\
\hline Cutoff radius $r_{c}$ & 1.6 \\
Mass density $\rho$ & 6.25 \\
Dynamic viscosity $\eta$ & 100 \\
Initial diameter of the grains $D_{0}$ & 8 \\
Superficial flow rate $q$ & 0.08 \\
Average number density $d_{0}$ & 6.25 \\
SDPD particle mass $m$ & 1 \\
Moment of inertial of SDPD particles $I$ & 1 \\
Size of the simulation domain $L_{x} \times L_{y}$ & $169.7 \times 186.7$ \\
Size of the sample region $L_{x} \times L_{s}$ & $169.7 \times 152.7$ \\
Number of grains $N_{g}$ & 225 \\
$P_{0}$ in the EoS & 25600 \\
Hydrostatic pressure $P_{0}-P_{\mathrm{b}}$ & 6400 \\
Exponent in the EoS $v$ & 7 \\
Spring force coefficient $k$ & 8000 \\
Break probability coefficient of the springs $p_{0}$ & 1.0 \\
Time step $\Delta t$ & 0.002 \\
Time step interval to perform the break action $N_{\text {freq }}$ & 10 \\
Bond number criterion $N_{b}$ & 7 \\
Temperature $k_{\mathrm{B}} T$ & $1 \mathrm{e}-6$ \\
\hline
\end{tabular}

throat size distribution can be determined through the procedure similar to that described by Chiu et a ${ }^{27}$ and de Anna et $a{ }^{54}$. First, a Delaunay triangulation is formed using the nodes placed at the mass center of each eroding grain. Then, all the edges $\overline{p_{i} q_{i}}$ are checked, if the closed disk with $\overline{p_{i} q_{i}}$ as a diameter contains any other vertices, this edge is deleted. The Gabriel graph ${ }^{55]}$ is obtained after all edges are examined. The neighboring grains are defined as those sharing an edge of the Gabriel graph. The pore throat sizes are finally determined by measuring the minimum distance between all pairs of neighboring grains. An example of applying this process to the initial configuration of a porous medium (Fig. 5 (b)) is shown in
Fig. 6 There are 225 grains in this porous medium. Because we are using periodic boundaries, we perform the Delaunay triangulation not only on these proper 225 grains, but also on all their periodic copies on the left and on the right. To avoid recounting some pores due to the existence of many copies of grains, only the pores among the proper 225 grains, and the pores between the proper grains and the copies on the right are counted to obtain the distribution of the pore sizes.

\section{E. Controlling the erosion rate}

As has been mentioned above, the erosion rate depends on three relevant parameters: shear erosion constant, which is of the order $C_{\mathrm{E}}^{\prime}$, cohesion parameter $c$, which is controlled by $\Delta l_{c}$, and the angle of internal friction $\psi$, which is fixed so far. Using the parameter given in Tab. I we have $C_{\mathrm{E}}^{\prime}=0.0025$. With $C_{\mathrm{E}}^{\prime}$, we can calculate the typical erosion time scale. First, a reference stress $\tau_{\text {ref }}=6 q \eta / \varepsilon_{0} / E_{0}(\lambda)=23.3$ is defined, where $\varepsilon_{0}=0.564$ is the initial porosity of the medium and $E_{0}(\lambda)=0.456 D_{0}$ is the initial mean pore size of the medium. This reference stress represents the shear stress at the boundary of a Poiseuille tube. The width of the tube is $E_{0}(\lambda)$ and the average flow rate inside the tube is $q / \varepsilon_{0}$. Then the typical time scale for a particle to be eroded is $t_{\text {er }}=m / \tau_{\text {ref }} / C_{\mathrm{E}}^{\prime}=17.17$. The typical time scale of erosion is also the time scale the solid boundary changes.

The parameters $D_{0}, q, \tau_{\text {ref }}, t_{\text {er }}$ will be used as reference parameters in the presentation of results, e.g. length $L^{*}=L / D_{0}$, velocity $V^{*}=V / q$, time $T^{*}=T / t_{\mathrm{er}}$, stress/pressure $P^{*}=$ $P / \tau_{\text {ref. }}$. Parameters marked with an asterisk are normalized.

Using the parameters in Tab. [1 and following Eq. (5) Eq. (9) we calculate the shear erosion threshold $\tau_{c}$ as a function of the applied normal stress $\sigma$ at different $\Delta l_{c}$. The results are shown in Fig. 7 Both $\tau_{c}$ and $\sigma$ are normalized with $\tau_{\text {ref }}$, 


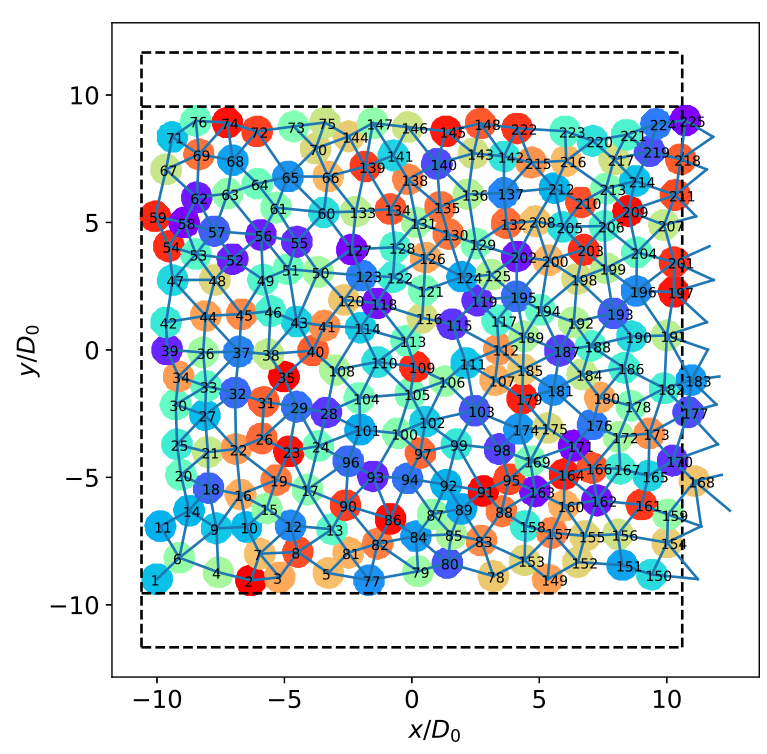

FIG. 6. Gabriel graph of the initial configuration of a porous medium. The 225 proper grains are displayed, while their periodic copies are not. The Gabriel graph is generated with the periodic copies included. The dash lines indicate the boundaries of the simulation box and the sample region. Some of the grains are outside the simulation box because we have rearranged some of the SDPD particles utilizing the periodic boundary condition to make sure all the grains are visually intact. The grains are colored for the purpose of distinguishing them from each other, there is no specific meaning for the colors.

the threshold $\Delta l_{c}$ is normalized using the average distance of the SDPD particles $\Delta x$. It can be seen that the larger the $\Delta l_{c}$ the higher the $\tau_{c}$. And the relationship between $\tau_{c}$ and $\sigma$ is always linear provided $\sigma$ is large enough (the slope of the failure envelope is 1 , i.e. the angle of internal friction is $\psi=\pi / 4$ ). Note that at the regime of small $\sigma$ and relatively large $\Delta l_{c}$, the slope is smaller than 1 , indicating that our model does not follow the Mohr-Coulomb yielding theory exactly. However, since we are only studying the problem qualitatively, Eq. (11) is adequate to describe our model. Therefore, we use the intercept value of $\tau_{c}$ as an approximation of the cohesion parameter $c$. From the figure the normalized cohesion parameters are $c^{*}=0.0,0.6,1.2,1.5$, and 1.8 for increasing $\Delta l_{c}$.

Since we did not introduce friction between particles the angle of internal friction arises solely from the competition between the normal force and tangential force. The normal force tends to compress the springs preventing them to break, while the tangential force tends to stretch the springs and break them. As long as the normal force and tangential force have the same effect on the springs (following the same Hooke's law) the angle of internal friction will always be $\psi=\pi / 4$. To tune the $\psi$, we need to find a way to control the contribution fraction of the normal force and tangential force on the springs. This can be done by including a scaling factor to the

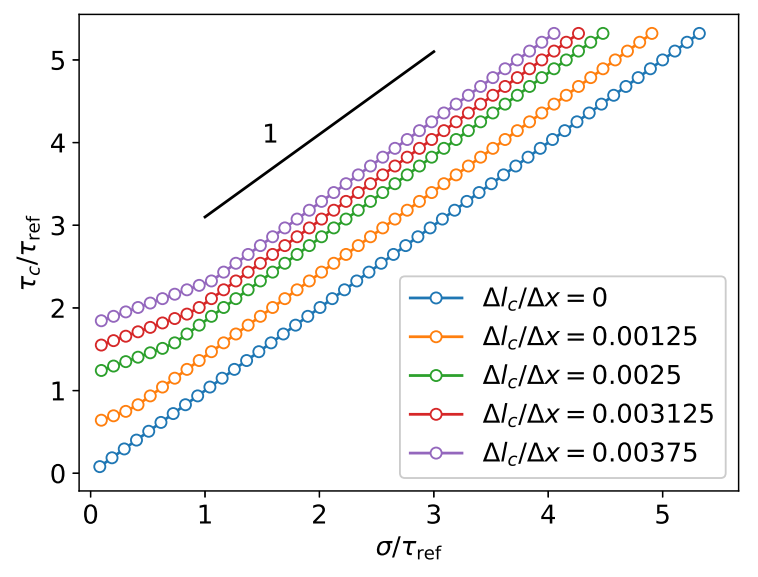

FIG. 7. Shear erosion threshold $\tau_{c}$ as a function of the applied normal stress $\sigma$ at different $\Delta l_{c} / \Delta x$. The values at $\sigma / \tau_{\text {ref }}=0$ are $c^{*} \equiv \tau_{c} / \tau_{\text {ref }}=0.0,0.6,1.2,1.5$, and 1.8 for increasing $\Delta l_{c} / \Delta x$.

conservative part of the pair-wise forces:

$$
\boldsymbol{F}_{i j}^{C}=\alpha\left(\frac{P_{i}}{d_{i}^{2}}+\frac{P_{j}}{d_{j}^{2}}\right) F_{i j} \boldsymbol{r}_{i j}, \quad i \in S
$$

where $\alpha>0$ is the scaling factor and $S$ is the set of the surface particles that are well supported (with at least $d$ links to internal particles, with $d$ being the dimension of the problem). Note that the inclusion of $\alpha$ has no direct effects on the fluid, it only affects the small displacement of the surface particles and their erosion rate. We also have

$$
\alpha=\tan \psi
$$

\section{RESULTS AND DISCUSSION}

In this part a typical eroding porous medium is first presented, the heterogeneity in the pressure drop direction, the pore sizes distribution, and flow rate distribution are discussed. Then we investigate the influence of the cohesion parameter and the angle of internal friction.

\section{A. Time dependent eroding geometry and characteristics}

We set the threshold $\Delta l_{c}=0, \alpha=1$ leading to $c=0$ and $\psi=\pi / 4$, the resultant eroding porous medium is presented in Fig. 8 . Figure. 8 (a)-(e) depict the eroding grains. As it can be seen the grains become smaller with the erosion progressing. At the bottom of the medium the erosion progresses significantly faster than at the top. This phenomenon is unique and cannot be observed in prior studies using simple shear-erosion mode 18227 . Obviously, this must be caused by the pressure dependence of the erosion rate in our model. As the flux is downward, the local pressure is much higher at the top than at 
(a)

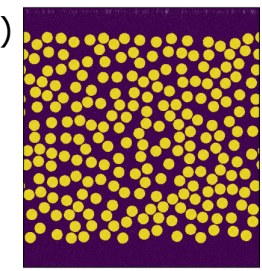

(f)

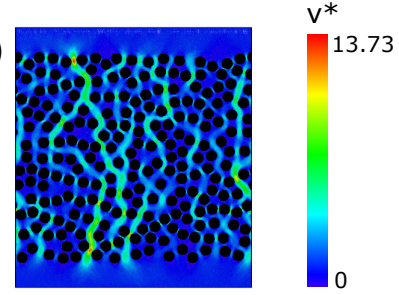

(b)

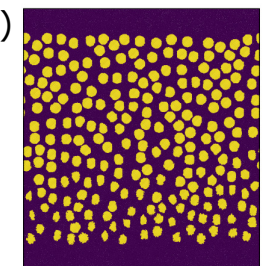

(c)

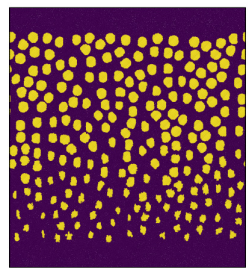

(d)

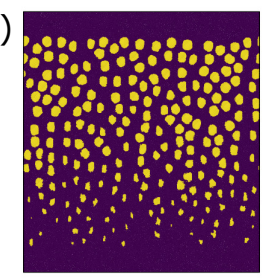

(e)

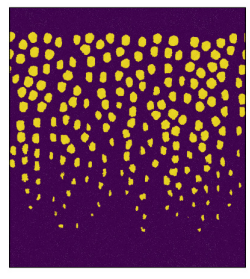

(g)

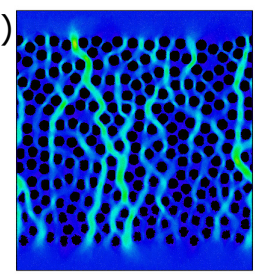

(h)

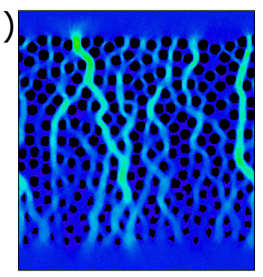

(i)

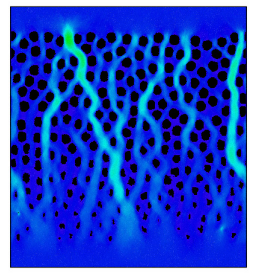

(j)

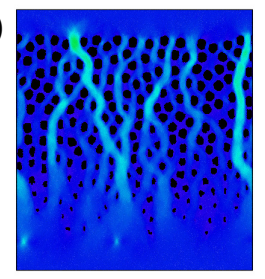

FIG. 8. Eroding porous medium. (a)-(e) depict the solid (grains) and the fluid. (a) $t^{*}=0$, (b) $t^{*}=2.33$, (c) $t^{*}=4.66$, (d) $t^{*}=6.99$, (e) $t^{*}=9.32$. (f)-(j) depict the corresponding velocity contour.

the bottom, and higher pressure tends to consolidate the grains and lowers the erosion rate, thus leading to the medium-scale heterogeneity in the filtering direction. Fig. 8(f)-(j) depict the evolution of the velocity contour. It can be seen that the flow is already inhomogeneous at the beginning. When the disks are placed randomly, some inter-grain pores are larger than others, forming some channels with higher hydraulic conductance. Since the flux in larger channels is generally larger, the erosion rate is also larger. With the erosion progressing, larger channels will increase further in size, causing more flow to be redirected to these channels. Eventually, this feedback effect can lead to an eroded geometry with just a few channels that transport a large portion of the flux ${ }^{27 / 56}$. The size of the channels is determined by the imbalance between the erosion rate in large channels and that in other small pores. In the simulation shown in Fig. 8, the channelization is not significant, and

we can see from (a)-(e) that there is not significant heterogeneity in the direction perpendicular to the filtration direction. This indicates that the erosion rate is not significantly different in different pores. Utilizing the fact that the eroding medium is horizontally relatively homogeneous, we divide the sample region with horizontal bins, and calculate the solid fraction $\phi$ and the average effective grain diameter $D$ in these bins, this gives us the $\phi-y$ and $D-y$ curves as shown in Fig. 9. Note that $D_{i}=2 \sqrt{A_{i} / \pi}$, with $A_{i}$ being the area of the $i$ th eroding grain. As it can be seen, at the beginning of the simulation the $\phi-y$ and $D-y$ curves are both relatively flat. With the erosion progressing, both curves become steeper, with the values at the lower $y$ endpoint decreasing faster than at the higher endpoint. At the end, a significant medium-scale heterogeneity in the direction of filtration is generated as a result of the very different local erosion rate.

Figure 10 shows the evolution of the pore sizes distribution. From (a) to (f) the porosity increases as a result of the erosion. Following the work of Chiu et $a^{[27}$ we fit the distribution using the Weilbull distribution $f\left(x ; \lambda^{\mathrm{wb}}, k^{\mathrm{wb}}\right)=$ $k^{\mathrm{wb}} / \lambda^{\mathrm{wb}}\left(x / \lambda^{\mathrm{wb}}\right)^{k^{\mathrm{wb}}-1} \exp \left(-\left(x / \lambda^{\mathrm{wb}}\right)^{k^{\mathrm{wb}}}\right)$. It can be seen that with the erosion progressing the distribution function becomes in better agreement with the Weilbull distribution. The agreement is the best when $\varepsilon=0.81$, then if the porosity continues increasing, the fit using the Weibull function becomes worse due to channelization. The fitted scale parameter $\lambda^{\mathrm{wb}}$ (given in the caption) is monotonically increasing. We show the mean and variance of pore sizes against the porosity in Fig. 11. As it can be seen the mean of the pore sizes increase steadily, while in the regime of small porosity, the variance of the pore sizes remains essentially the same. Therefore, when the porosity is relatively small channelization is less prevalent. These results are in good agreement with the simulations of Chiu et a 27 , even though here we are using a pressuredependent erosion model.

The local flow rate in the porous medium can range over several orders of magnitude. It has been revealed that, at low flow rate regime, the probability density function (PDF) of flow rate could be determined by the pore size distribution ${ }^{54}$. On the other hand, at high flow rate regime, it is the correlation between adjacent pores that determines the flow rate distribution [57]. High disorder leads to exponential decay of the PDF of flow rate ${ }^{54 / 57 / 58}$. Figure 12 shows the PDF of the flow rate at different instant in one simulation. It can be seen that the PDF decays exponentially at the beginning, whereas, with the erosion progressing, deviations occur at the very high flow rate regime. A peak is also induced near the $v / \bar{v}=1$.

\section{B. Effects of the cohesion parameter}

Using effective continuum multiphase model Derr et a ${ }^{21}$ have revealed that channel width is mainly determined by the correlation length of the initial condition, and how fast the flows are redirected compared to the drop of erosion threshold over a stress communication length. Using "shear erosion" model to perform LBM simulation of eroding porous medium, Jäger et $a l^{[25}$ found that the larger the range of wall shear 
(a)

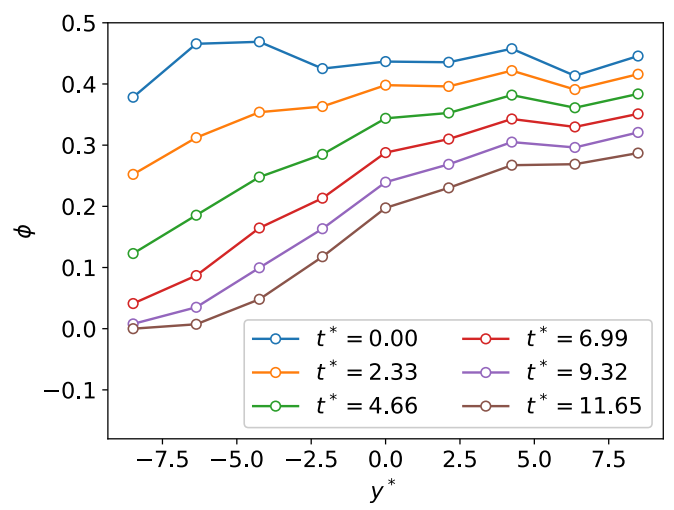

(b)

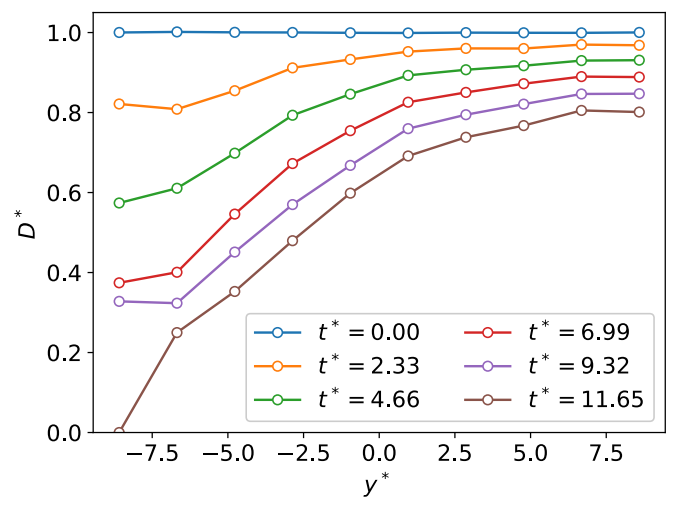

FIG. 9. (a) Solid fraction $\phi$ as a function of vertical position $y^{*}$. (b) Effective grains diameter $D^{*}$ as a function of the vertical position. The lines connecting the data points are only for guidance of eye.

(a)

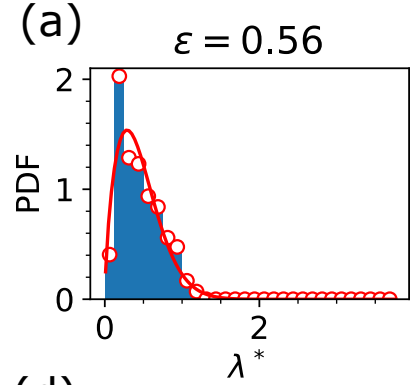

(d)

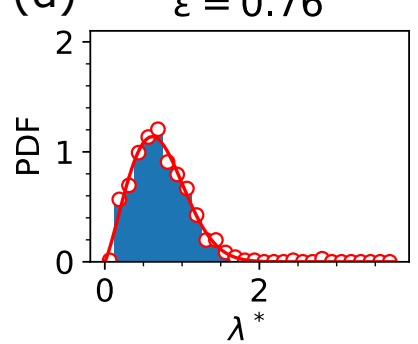

(b)

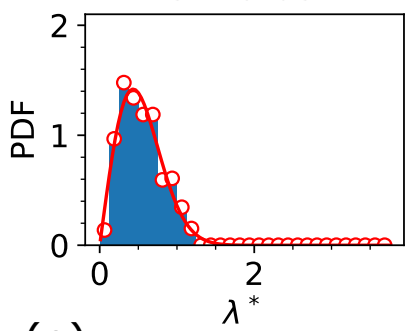

(e) $\varepsilon=0.81$

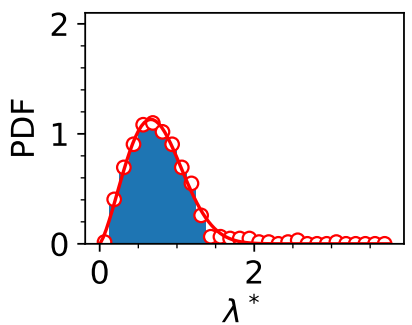

(c)

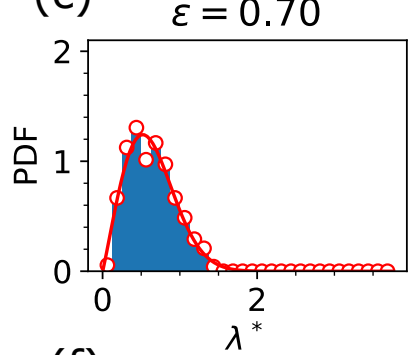

(f) $\varepsilon=0.84$

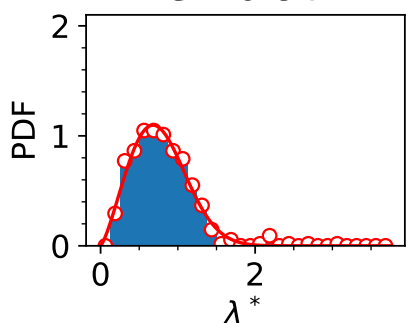

FIG. 10. Pore sizes distribution at different porosity. $\lambda^{*}$ is the dimensionless pore size, $\varepsilon$ is the porosity, the red lines are fits using the Weilbull distribution with parameter $\left(k^{\mathrm{wb}}, \lambda^{\mathrm{wb}}\right)$. (a) $t^{*}=0,\left(k^{\mathrm{wb}}, \lambda^{\mathrm{wb}}\right)=(1.66,0.50) ;(\mathrm{b}) t^{*}=2.33,\left(k^{\mathrm{wb}}, \lambda^{\mathrm{wb}}\right)=(2.02,0.61) ;(\mathrm{c}) t^{*}=4.66$, $\left(k^{\mathrm{wb}}, \lambda^{\mathrm{wb}}\right)=(2.09,0.71) ;(\mathrm{d}) t^{*}=6.99,\left(k^{\mathrm{wb}}, \lambda^{\mathrm{wb}}\right)=(2.21,0.81) ;(\mathrm{e}) t^{*}=9.32,\left(k^{\mathrm{wb}}, \lambda^{\mathrm{wb}}\right)=(2.33,0.84) ;(\mathrm{f}) t^{*}=11.65,\left(k^{\mathrm{wb}}, \lambda^{\mathrm{wb}}\right)=$ $(2.32,0.87)$.

stress for which there is neither erosion nor deposition, the more steady channels are formed in the structure. Also with "shear erosion" model but using boundary integral method, Chiu et a ${ }^{27}$ analyze the porosity-tortuosity relationship, the asysmptotic dispersion rate, and the pore-size distribution during erosion process. However, it has not been investigated how the failure properties of the grains affect the channelization. We see above that channelization is less prevalent when the cohesion parameter $c=0$. Here we examine the effects of the $c$ on channelization and other porous characteristics.

Fig. 13 shows how different the eroding porous media are when all the porous media reach the same target porosity $\varepsilon=0.84$. From (a)\&(b) to (i) $\&(\mathrm{j})$ the cohesion parameter $c^{*}$ is increased from 0 to 1.8 . It can be seen that channelization is more and more significant with the increase of the cohesion. When $c^{*} \geq 1.5$ two main channels are formed with nearly all flow redirected here. This phenomenon can be explained as follows. Larger $c^{*}$ means that the grains become harder to yield, and in smaller pores the local flow rate is more likely to be too small to erode the grains. Erosion occurs therefore predominantly in the larger pores and this facilitates the redirection of flow to those faster eroding pores, thus enhancing channelization.

The solid fraction and average effective grain diameter as a function of vertical position is presented in Fig. 14 (a) and (b). Here the curves in (a) and (b) corresponds to the five sets 
(a)

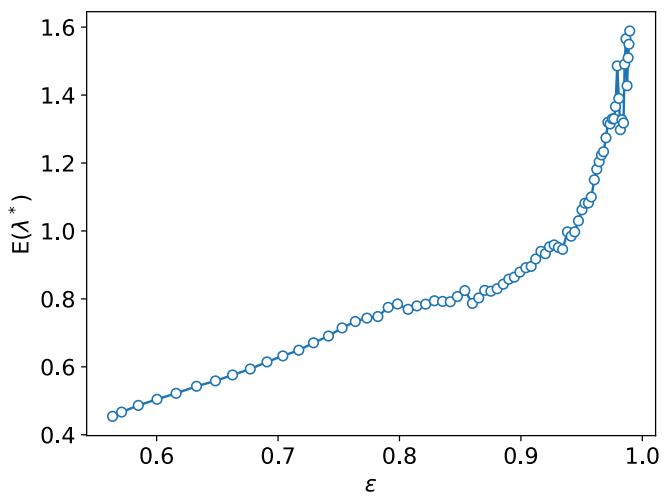

FIG. 11. (a) Mean of the pore sizes as a function of the porosity. connecting the data points are only for guidance of eye.

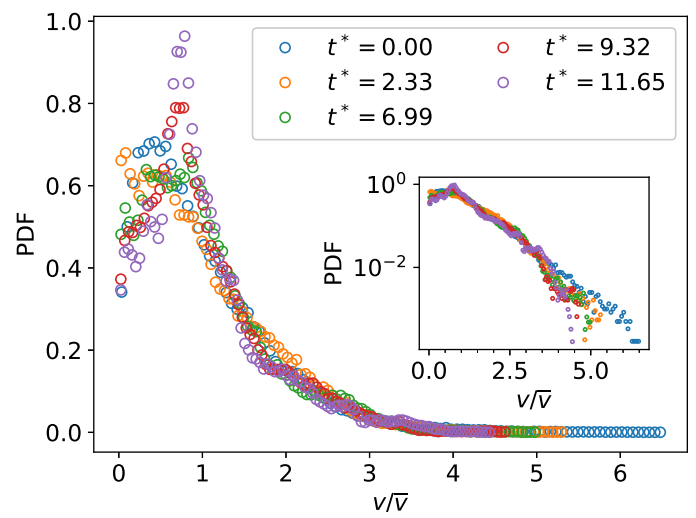

FIG. 12. Probability density function of the flow rate at different instant in one simulation. The 5 instants correspond to the 5 snapshots in Fig. 8. Inlet shows the same data on semilogarithmic axes.

of results in Fig. 13 Interestingly, the curve of solid fraction is only slightly affected by the variation of $c^{*}$, but the curve of effective grain diameter is significantly affected. Larger $c^{*}$ leads to greater effective diameter, especially at the center part of the medium. This phenomenon is also evident in Fig. 13. These suggest that the medium-scale heterogeneity induced by the pressure dependence of the erosion is not affected by systematically varying the cohesion parameter, while the distribution of the diameter of the eroding grains will be significantly affected.

Figure 15 (a) and (b) show the means and the variances of pore throat sizes against porosity at different cohesion parameter $c^{*}$. It can be seen that the means of the pore sizes at different $c^{*}$ almost fall onto one curve, while the variances of the pore size are the same for different $c^{*}$ only at relatively small porosity. At higher porosity, the local flow rate becomes small enough so that at least some of the small pores will cease erosion because of the yield threshold. Then the flow is redirected, and larger pores are eroded faster. This explains the (b)

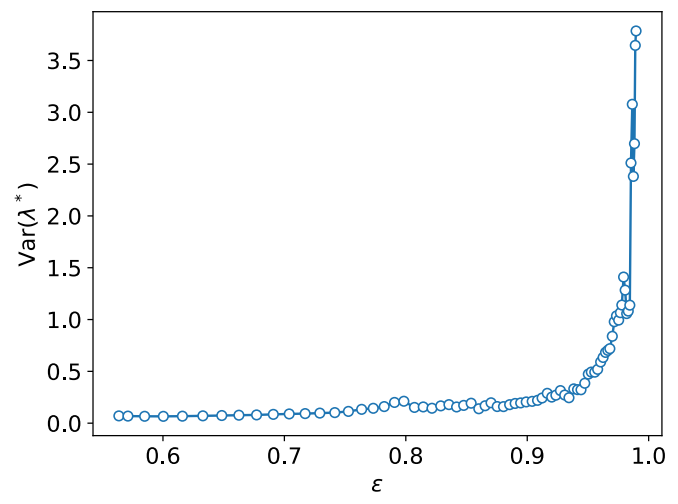

(b) Variance of the pore sizes as a function of the porosity. The lines

large variance in the regime of higher $\varepsilon$. Moreover, the larger the cohesion parameter $c^{*}$ the earlier the actual stress becomes smaller than the yield threshold, and this leads to larger variance for larger $c^{*}$ at the same $\varepsilon$.

\section{Effects of the angle of internal friction}

The angle of internal friction is controlled by the scaling factor $\alpha$ following Eq. (17). Here we vary $\tan \psi$ to examine the effects of the angle of internal friction.

Figure 16 shows eroding porous media with $\tan \psi$ varied from 0 to 1. Erosion in these porous media start with the same initial condition. With the erosion progressing they will all reach the same target porosity $\varepsilon=0.81$ as shown in the figure. It can be seen that the effects of the angle of internal friction is quite significant: larger $\tan \psi$ results in higher degree of heterogeneity in the filtration direction but lower degree of heterogeneity in the transverse direction. The effects on the heterogeneity in the filtration direction are also manifested in Fig. 17 It can be seen from Fig. 17 that larger $\tan \psi$ leads to steeper spatial distribution of both solid fraction $\phi$ and effective grain diameter $D$ in the filtration direction. This is obviously due to the fact that $\tan \psi$ controls the pressure dependence of the erosion. Larger $\tan \psi$ means the erosion is more pressure-dependent, inducing higher heterogeneity in the pressure drop direction. When $\tan \psi=0$ the erosion is essentially pressure-independent, then there will be no heterogeneity in the pressure drop direction as demonstrated in Fig. 17 .

Inspecting Fig. 16 , channelization (heterogeneity in the transverse direction) is impeded by increasing $\tan \psi$. This is probably caused by the combined effects of two factors. One is the fact that the channelization is only prevalent when the porosity is relatively high 27 . The other is the flow correlation in the filtration direction. The mechanics of the $\tan \psi$ affecting channelization can be explained as follows. Larger $\tan \psi$ induces steeper distribution of porosity in the filtration direction (see Fig. 17](a)), the porosity is thus lower at the upstream 
(a)

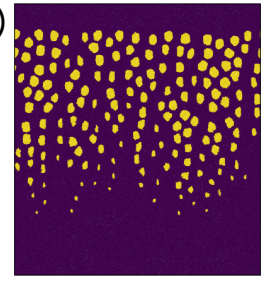

(b)

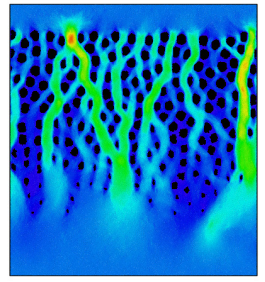

(c)

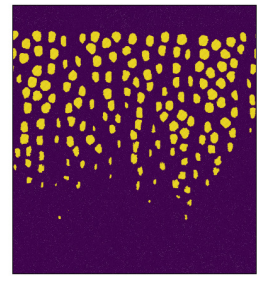

(d)

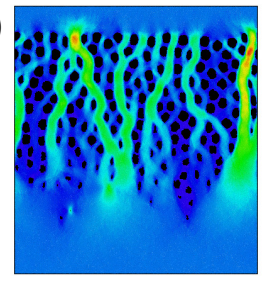

(e)

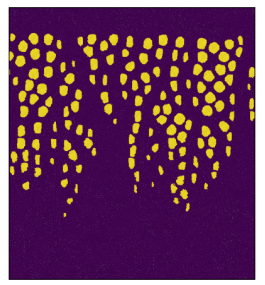

(f)

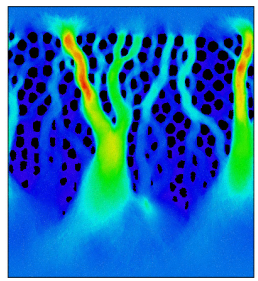

(g)

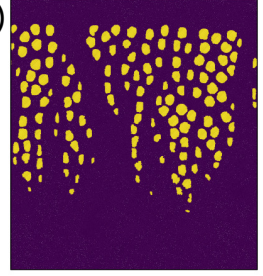

(h)

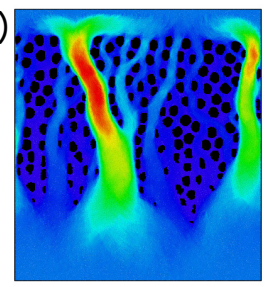

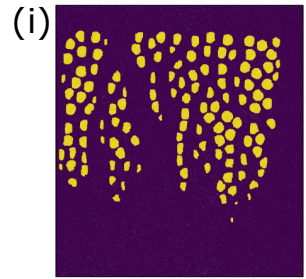

(j)

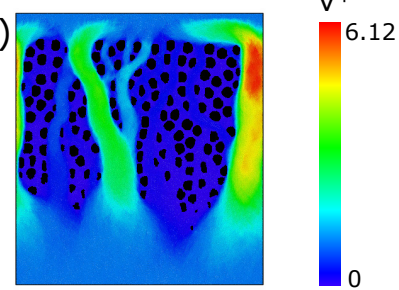

FIG. 13. Eroding porous media with different cohesion. (a)-(b) $c^{*}=0$, (c)-(d) $c^{*}=0.6$, (e)-(f) $c^{*}=1.2$, (g)-(h) $c^{*}=1.5$, (i)-(j) $c^{*}=1.8$. (a) (c) (e) (g) and (i) depict eroding grains and the fluid, (b) (d) (f) (h) and (j) depict the corresponding velocity contours. Porosity is $\varepsilon=0.84$ for all results demonstrated.

(a)

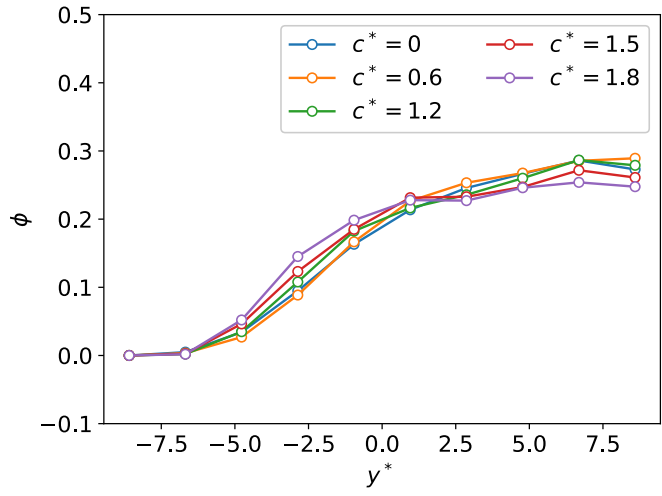

(b)

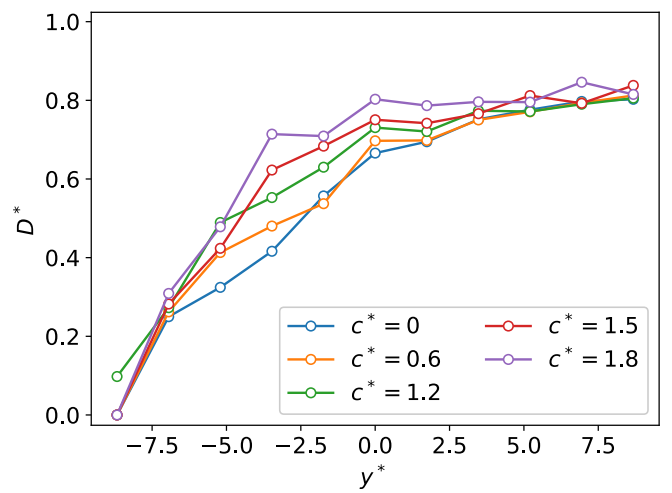

FIG. 14. (a) Solid fraction as a function of the vertical position at different cohesion $c^{*}$. (b) Average effective grain diameter as a function of the vertical position at different cohesion $c^{*}$. For all curves in (a) and (b) $\varepsilon=0.84$. The lines connecting the data points are only for guidance of eye.

(a)

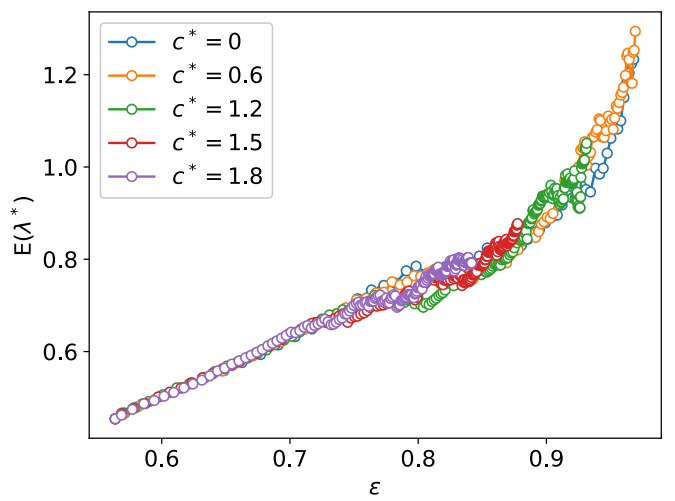

(b)

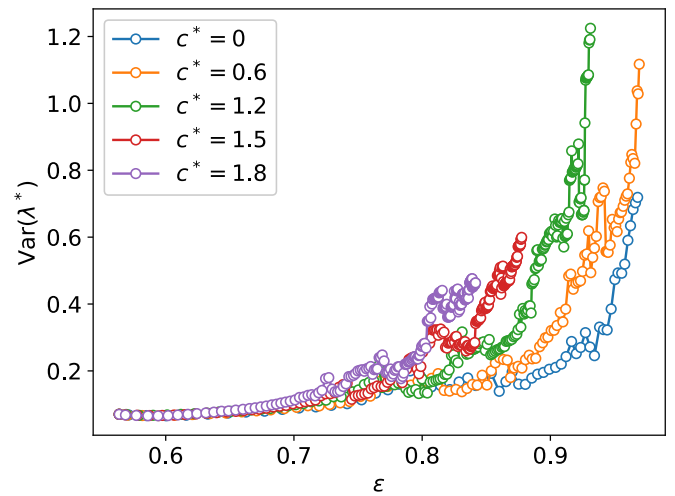

FIG. 15. (a) Mean of the pore sizes as a function of the porosity at different $c^{*}$. (b) Variance of the pore sizes as a function of the porosity at different $c^{*}$. The lines connecting the data points are only for guidance of eye.

part leading to less or no channelization there. The channel- ization is just the result of flows redirection due to different 
(a)

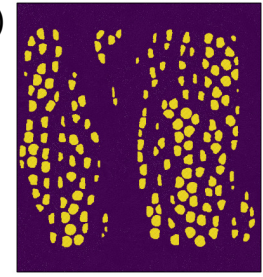

(b)

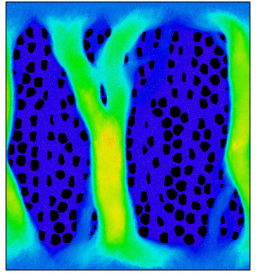

(c)

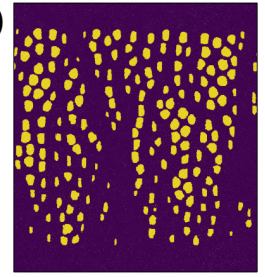

(d)

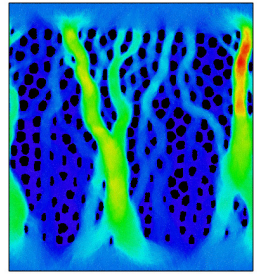

(e)

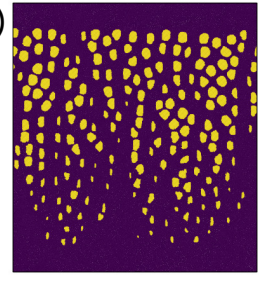

(f)

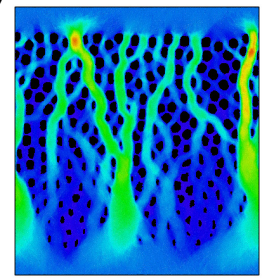

(g)

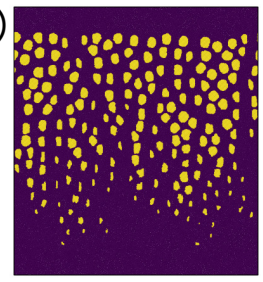

(h)

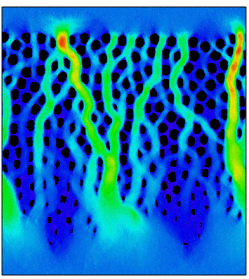

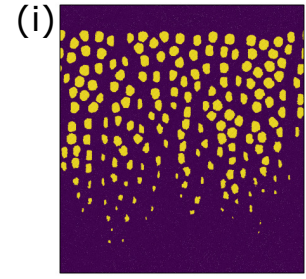

(j)

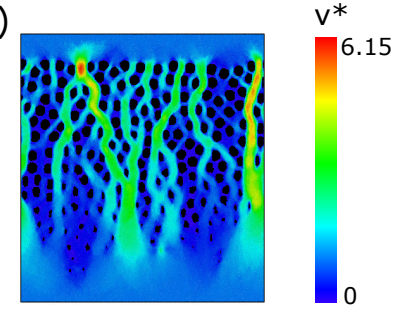

FIG. 16. Eroding porous media with different angle of internal friction. (a)-(b) $\tan \psi=0$, (c)-(d) $\tan \psi=0.25$, (e)-(f) $\tan \psi=0.5$, (g)-(h) $\tan \psi=0.75$, (i)-(j) $\tan \psi=1.0$. (a) (c) (e) (g) and (i) depict eroding grains and the fluid, (b) (d) (f) (h) and (j) depict the corresponding velocity contours. Porosity is $\varepsilon=0.81$ for all results demonstrated.

hydraulic conductance. If the channelization at the upstream part is suppressed, it means that flows there stay dispersed. Then these dispersed upstream flows will also suppress the flow redirection at the downstream part, leading to insignificant channelization overall.

Figure 18 (a) and (b) show the means and the variances of pore throat sizes against porosity at different $\tan \psi$. It can be seen that at the regime of relatively small porosity the change of both the mean and variance of the pore throat sizes follows the same trajectories. Only at the high porosity regime the effects of $\tan \psi$ manifest. Unlike the cohesion parameter $c$ which affects only the variance of the pore throat sizes, the angle of internal friction $\psi$ has significant effects on both the mean and the variance. Both the mean and the variance tend to be smaller if $\tan \psi$ is larger. Obviously, the decrease of variance of the pore throat sizes is the result of the suppression of channelization caused by higher $\tan \psi$.

\section{SUMMARY AND CONCLUSION}

In our previous work ${ }^{16}$ we have incorporated the double (liquid/intra-granular) molecular diffusion, solid-liquid release mechanism, and the migration of fines (cellular fragments) in a particle-based model to simulate the espresso extraction. In the present work, we focus on the geometric change of the coffee cake induced by hydrodynamic forces. Based on prior experimental observation ${ }^{37}$, we assume that the coffee grains are not completely wetted and remain sufficiently brittle. We devise a bottom-up mechanical erosion model, which can be seen as a combination of the MohrCoulomb yield criteria and classic shear-erosion model, to investigate eroding coffee cake. Using the present model, we found that an eroding porous medium whose yield threshold is pressure-dependent has a noteworthy feature: heterogeneity in both the filtration direction and the direction perpendicular to it can be induced. The former is caused mainly by the pressure difference across the porous medium, the latter, which is also known as "channelization", is caused by the positive feedback of hydraulic conductance increase induced by erosion. The medium-scale heterogeneity in the filtration direction, which is manifested by the spatial distribution of porosity, is mainly controlled by the angle of internal friction of the material $\psi$. It is barely affected by the cohesion parameter. Higher value of $\psi$ leads to more significant heterogeneity. On the other hand, the heterogeneity in the transverse direction, which is manifested by the variance of the pore throat sizes and the large channels seen after erosion, can be controlled by both the cohesion parameter $c$ and the angle of internal friction $\psi$. The higher the $c$ the more significant the channelization will be, provided that the external hydrodynamics is strong enough to erode the medium. The larger the $\psi$ the less significant the channelization is, because the heterogeneity in the filtration direction tends to suppress channelization.

To summarize, we have studied, in this paper, how the Mohr-Coulomb parameters affect the erosion of a porous medium. There are plenty of studies $\frac{18[25 \llbracket 27}{20}$ fousing on modelling eroding porous medium with the simple "shear erosion" criterion. The Mohr-Coulomb criterion (or similar criterion e.g. Drucker-Prager) has also already been incorporated into erosion model in simulations of sediment erosior $30+32$. But to the best of our knowledge, the effects of applying the MohrCoulomb criterion to eroding porous medium has not been studied. This highlights the novelty of our research.

Even though the main motivation of this research is connected to the modeling of espresso extraction, the erosion model we have devised and the obtained results can be applied to other eroding porous media. Our results suggest that the heterogeneity of an eroding medium (whose failure criterion is pressure-dependent) can be controlled by tuning the yield characteristics of eroding material (e.g. by controlling the temperature or water content of the material).

As discussed in the introduction, the effects of pressuredependent erosion on the time-dependent mass distribution in 
(a)

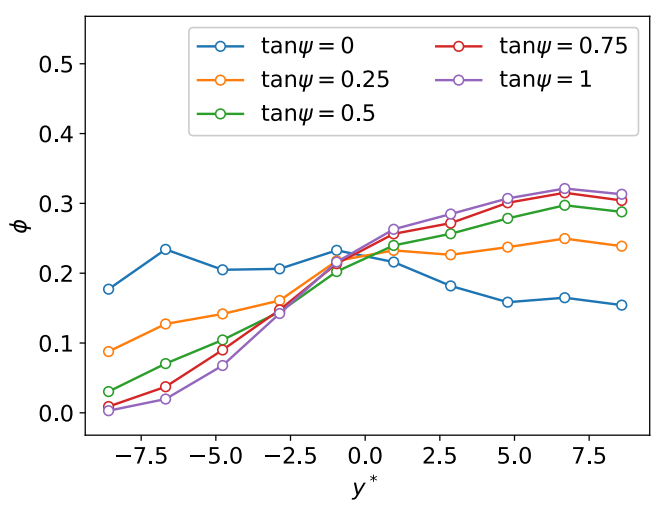

(b)

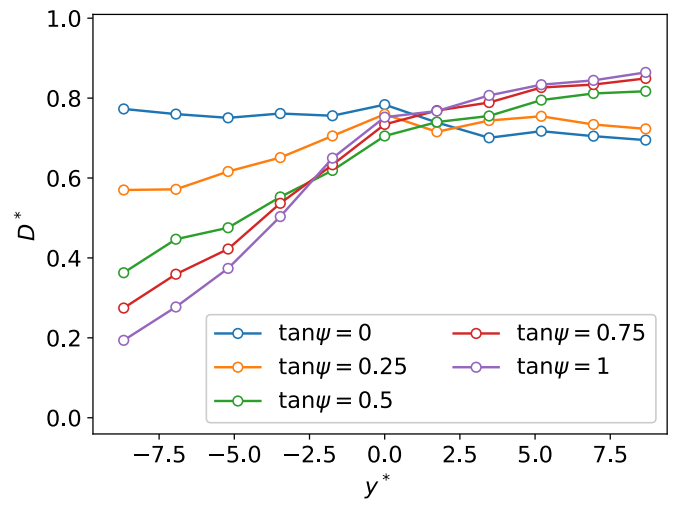

FIG. 17. (a) Solid fraction as a function of the vertical position at different tan $\psi$. (b) Average effective grain diameter as a function of the vertical position at different $\tan \psi$. For all curves in (a) and (b) $\varepsilon=0.81$. The lines connecting the data points are only for guidance of eye.

(a)

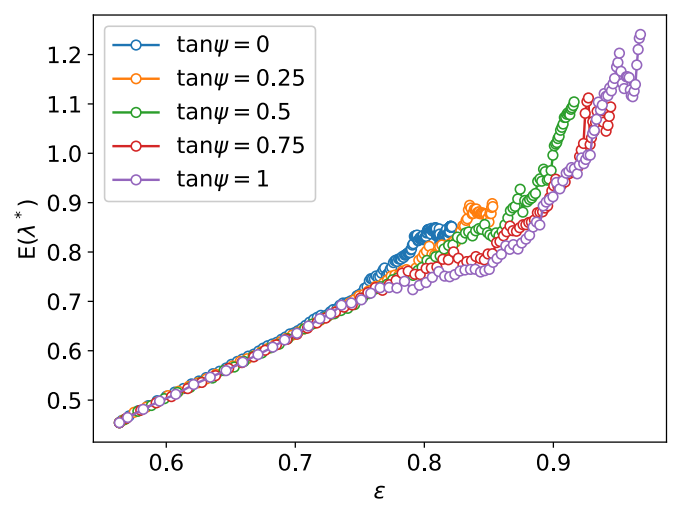

(b)

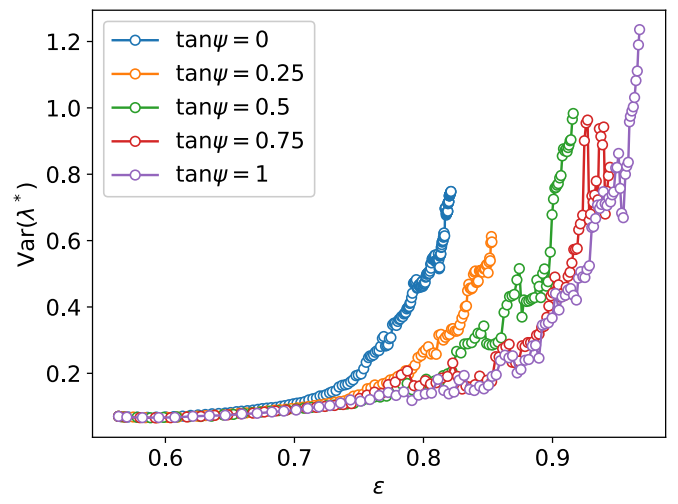

FIG. 18. (a) Mean of the pore sizes as a function of the porosity at different tan $\psi$. (b) Variance of the pore sizes as a function of the porosity at different $\tan \psi$. The lines connecting the data points are only for guidance of eye.

a porous medium is only one of the many important physical processes occurring inside a coffee cake during extraction. Other highly-relevant effects are related to consolidation and compaction $\frac{5960}{}$, fragment migrations ${ }^{416}$ and grain swelling 6162 , to mention but a few. To further improve the mesoscopic modeling of espresso extraction in the future we plan to model the coffee grains as quasi-rigid body to consider both the consolidation and fracture of the grains. Performing $\mathrm{X}$-ray microCT scans on both dry coffee cake and coffee cake after extraction is also crucial to gather more microstructural information and this is indeed the object of current investigations. X-ray microCT is a non-destructive imaging technique that can provide the 3D geometry of the porous structure. The solid structure can be segmented from the pores, providing a real structure for porous media modeling studies ${ }^{63164}$, in addition to visualisation and analysis of temporal changes in the structure. It is expected that analysis on the microCT images could provide crucial information about how the geometry changes during extraction process. This information will provide important guidance to disentangle the effects discussed above and to devise appropriate simulation techniques that can incorporate all the relevant dynamics (erosion, consolidation swelling, and fines migration) into the espresso extraction simulation, i.e. moving towards more quantitative virtual coffee profiling.

\section{ACKNOWLEDGEMENT}

This research is supported by the Illycaffe' S.p.a. through the project "Modelling and simulating espresso coffee extraction at the mesoscopic scales". Financial support from grant RTI2018-094595-B-I00 and BCAM Severo Ochoa excellence accreditation SEV-2017-0718 funded by (AEI/FEDER, UE) are also acknowledged. 


\section{DATA AVAILABILITY}

The data that support the findings of this study are available from the corresponding author upon reasonable request.

\section{BIBLIOGRAPHY}

${ }^{1}$ Ernesto Illy. The complexity of coffee. Scientific American, 286(6):86-91, 2002.

${ }^{2}$ James Hoffmann. The world atlas of coffee: From beans to brewing. Firefly Books, 2014.

${ }^{3}$ Michael I. Cameron, Dechen Morisco, Daniel Hofstetter, Erol Uman, Justin Wilkinson, Zachary C. Kennedy, Sean A. Fontenot, William T. Lee, Christopher H. Hendon, and Jamie M. Foster. Systematically improving espresso: Insights from mathematical modeling and experiment. Matter, 2(3):631-648, 2020.

${ }^{4} \mathrm{M}$ Petracco and F Suggi Liverani. Espresso coffee brewing dynamics: development of mathematical and computational models. In Colloque Scientifique International sur le Cafe, volume 15, pages 702-702. ASIC ASSOCIATION SCIENTIFIQUE INTERNATIONALE, 1993.

${ }^{5}$ A Voilley and D Simatos. Modeling the solubilization process during coffee brewing. Journal of Food Process Engineering, 3(4):185-198, 1980.

${ }^{6}$ Andrea Illy and Rinantonio Viani. Espresso coffee: the science of quality. Academic Press, 2005.

${ }^{7}$ Ernesto Illy and Luciano Navarini. Neglected food bubbles: the espresso coffee foam. Food biophysics, 6(3):335-348, 2011.

${ }^{8}$ John R Melrose. Part i: Internal coffee particle phases and coffee brewing release profiles. In Physics in Food Manufacturing, 2053-2563, pages 5-1 to 5-18. IOP Publishing, 2020.

${ }^{9}$ John R Melrose. Part ii: Modelling coffee brewing release profiles. In Physics in Food Manufacturing, 2053-2563, pages 6-1 to 6-24. IOP Publishing, 2020.

${ }^{10}$ Federico Talamucci. Flow through a porous medium with mass removal and diffusion. Nonlinear Differential Equations and Applications NoDEA, 5(4):427-444, Nov 1998.

${ }^{11}$ A. Fasano and F. Talamucci. A comprehensive mathematical model for a multispecies flow through ground coffee. SIAM Journal on Mathematical Analysis, 31(2):251-273, 2000

${ }^{12}$ Kevin M. Moroney, William T. Lee, Stephen BG Brien, Freek Suijver, and Johan Marra. Coffee extraction kinetics in a well mixed system. Journal of Mathematics in Industry, 7(1):3, Jun 2016.

${ }^{13}$ K.M. Moroney, W.T. Lee, S.B.G. O' Brien, F. Suijver, and J. Marra. Modelling of coffee extraction during brewing using multiscale methods: An experimentally validated model. Chemical Engineering Science, 137:216234, 2015.

${ }^{14}$ Kevin M. Moroney, K O'Connell, P Meikle-Janney, Stephen BG Brien, GM Walker, and William T. Lee. Analysing extraction uniformity from porous coffee beds using mathematical modelling and computational fluid dynamics approaches. PLoS ONE, 14(7):1-24, 2019.

${ }^{15}$ David Beverly, Estefanía Lopez-Quiroga, Robert Farr, John Melrose, Sian Henson, Serafim Bakalis, and Peter J. Fryer. Modeling Mass and Heat Transfer in Multiphase Coffee Aroma Extraction. Industrial and Engineering Chemistry Research, 59(24):11099-11112, 2020.

${ }^{16} \mathrm{M}$. Ellero and L. Navarini. Mesoscopic modelling and simulation of espresso coffee extraction. Journal of Food Engineering, 263(May):181194, 2019.

${ }^{17}$ R. H. Rosa, G. V. Von Atzingen, V. Belandria, A. L. Oliveira, S. Bostyn, and J. A. Rabi. Lattice Boltzmann simulation of cafestol and kahweol extraction from green coffee beans in high-pressure system. Journal of Food Engineering, 176:88-96, 2016.

18 André F.V. Matias, Rodrigo C.V. Coelho, José S. Andrade, and Nuno A.M. Araújo. Flow through time-evolving porous media: Swelling and erosion. Journal of Computational Science, 53(Jan 2021), 2021.

${ }^{19}$ Norbert Schorghofer, Bill Jensen, Arshad Kudrolli, and Daniel H. Rothman. Spontaneous channelization in permeable ground: Theory, experiment, and observation. Journal of Fluid Mechanics, 503(503):357-374, 2004.
${ }^{20}$ Michael C. Malin and Kenneth S. Edgett. Evidence for recent groundwater seepage and surface runoff on mars. Science, 288(5475):2330-2335, 2000.

${ }^{21}$ Nicholas J Derr, David C Fronk, Christoph A Weber, Amala Mahadevan, Chris H Rycroft, and L Mahadevan. Flow-Driven Branching in a Frangible Porous Medium. Physical Review Letters, 125(15):158002, 2020.

${ }^{22}$ A. Mahadevan, A. V. Orpe, A. Kudrolli, and L. Mahadevan. Flow-induced channelization in a porous medium. Epl, 98(5), 2012.

${ }^{23}$ Braunen Smith, Arshad Kudrolli, Alexander E. Lobkovsky, and Daniel H. Rothman. Channel erosion due to subsurface flow. Chaos: An Interdisciplinary Journal of Nonlinear Science, 18(4):041105, 2008.

${ }^{24}$ Arshad Kudrolli and Xavier Clotet. Evolution of Porosity and Channelization of an Erosive Medium Driven by Fluid Flow. Physical Review Letters, 117(2):1-5, 2016

${ }^{25}$ R. Jäger, M. Mendoza, and H. J. Herrmann. Channelization in porous media driven by erosion and deposition. Physical Review E, 95(1):1-11, 2017.

${ }^{26}$ R. Jäger, M. Mendoza, and H. J. Herrmann. Mechanism behind Erosive Bursts in Porous Media. Physical Review Letters, 119(12):1-5, 2017.

${ }^{27}$ Shang Huan Chiu, M. N.J. Moore, and Bryan Quaife. Viscous transport in eroding porous media. Journal of Fluid Mechanics, 893, 2020.

${ }^{28}$ Leif Ristroph, Matthew N.J. Moore, Stephen Childress, Michael J. Shelley, and Jun Zhang. Sculpting of an erodible body by flowing water. Proceedings of the National Academy of Sciences of the United States of America, 109(48):19606-19609, 2012.

${ }^{29}$ Matthew N.J. Moore, Leif Ristroph, Stephen Childress, Jun Zhang, and Michael J. Shelley. Self-similar evolution of a body eroding in a fluid flow. Physics of Fluids, 25(11), 2013.

${ }^{30}$ Elizabeth H. Zubeldia, Georgios Fourtakas, Benedict D. Rogers, and Márcio M. Farias. Multi-phase SPH model for simulation of erosion and scouring by means of the shields and Drucker-Prager criteria. Advances in Water Resources, 117(December 2017):98-114, 2018.

${ }^{31}$ G. Fourtakas and B. D. Rogers. Modelling multi-phase liquid-sediment scour and resuspension induced by rapid flows using Smoothed Particle Hydrodynamics (SPH) accelerated with a Graphics Processing Unit (GPU). Advances in Water Resources, 92:186-199, 2016.

${ }^{32}$ Sauro Manenti, Stefano Sibilla, Mario Gallati, Giordano Agate, and Roberto Guandalini. Sph simulation of sediment flushing induced by a rapid water flow. Journal of Hydraulic Engineering, 138(3):272-284, 2012.

${ }^{33}$ Megan J Povey, Elena Simone, Melvin J Holmes, Michael Rappolt, Sameera Rafiq, and Mat Francis. Physics in Food Manufacturing. IOP Publishing, Bristol, UK, 2020.

${ }^{34}$ Britta Folmer. The craft and science of coffee. Academic Press, 2016.

${ }^{35}$ P. Pittia, M. Dalla Rosa, and C.R. Lerici. Textural changes of coffee beans as affected by roasting conditions. LWT - Food Science and Technology, 34(3):168-175, 2001

${ }^{36} \mathrm{O}$ Fond. Effect of water and coffee acidity on extraction. dynamics of coffee bed compaction in espresso type extraction. In Proceedings of the 16th International Scientific Colloquium on Coffee, ASIC (Kyoto).(ASIC, Paris, 1995) pp, volume 413420, 1995.

${ }^{37}$ Maria L. Mateus, Martine Rouvet, Jean C. Gumy, and Rémy Liardon. Interactions of water with roasted and ground coffee in the wetting process investigated by a combination of physical determinations. Journal of Agricultural and Food Chemistry, 55(8):2979-2984, 2007.

${ }^{38}$ Richard M. Christensen. The Theory of Materials Failure. Oxford University Press, 2013.

${ }^{39}$ Joseph F Labuz and Arno Zang. Mohr-coulomb failure criterion. In The ISRM Suggested Methods for Rock Characterization, Testing and Monitoring: 2007-2014, pages 227-231. Springer, 2012.

${ }^{40}$ P. Español and M. Revenga. Smoothed dissipative particle dynamics. Phys. Rev. E, 67:026705, 2003.

${ }^{41}$ K. Müller, D. A. Fedosov, and G. Gompper. Smoothed dissipative particle dynamics with angular momentum conservation. J. Comp. Phys., 281:301315, 2015.

${ }^{42} \mathrm{M}$ Ellero and $\mathrm{P}$ Español. Everything you always wanted to know about sdpd (but were afraid to ask). Applied Mathematics and Mechanics, 39(1):103124, 2018.

${ }^{43}$ J. J. Monaghan. Smoothed particle hydrodynamics. Annu. Rev. Astron. Astrophys., 30:543-574, 1992.

${ }^{44}$ P. J. Hoogerbrugge and J. M. V. A. Koelman. Simulating microscopic hydrodynamic phenomena with dissipative particle dynamics. Europhys. Lett., 19:155-160, 1992. 
${ }^{45} \mathrm{P}$. Español and P. Warren. Statistical mechanics of dissipative particle dynamics. Europhys. Lett., 30:191-196, 1995.

${ }^{46}$ Adolfo Vázquez-Quesada, Marco Ellero, and Pep Español. Consistent scaling of thermal fluctuations in smoothed dissipative particle dynamics. The Journal of Chemical Physics, 130(3):034901, 2009.

${ }^{47}$ X. Y. Hu and N. A. Adams. Angular-momentum conservative smoothed particle dynamics for incompressible viscous flows. Phys. Fluids, 18:101702, 2006.

${ }^{48}$ I. O. Götze, H. Noguchi, and G. Gompper. Relevance of angular momentum conservation in mesoscale hydrodynamics simulations. Phys. Rev. E, 76:046705, 2007.

${ }^{49}$ M. P. Allen and D. J. Tildesley. Computer simulation of liquids. Clarendon Press, New York, 1991.

${ }^{50}$ L. B. Lucy. A numerical approach to the testing the fission hypothesis. Astronom. J., 82:1013-1024, 1977.

${ }^{51}$ Navid Bizmark, Joanna Schneider, Rodney D. Priestley, and Sujit S. Datta. Multiscale dynamics of colloidal deposition and erosion in porous media. Science advances, 6(46): 1-11, 2020.

${ }^{52}$ William H. Mitchell and Saverio E. Spagnolie. A generalized traction integral equation for Stokes flow, with applications to near-wall particle mobility and viscous erosion. Journal of Computational Physics, 333:462-482, 2017.

${ }^{53}$ Qihua Ran, Jian Tong, Songdong Shao, Xudong Fu, and Yueping Xu. Incompressible SPH scour model for movable bed dam break flows. Advances in Water Resources, 82:39-50, 2015.

${ }^{54}$ Pietro de Anna, Bryan Quaife, George Biros, and Ruben Juanes. Prediction of the low-velocity distribution from the pore structure in simple porous media. Phys. Rev. Fluids, 2:124103, Dec 2017.
${ }^{55}$ K. Ruben Gabriel and Robert R. Sokal. A New Statistical Approach to Geographic Variation Analysis. Systematic Biology, 18(3):259-278, 09 1969.

${ }^{56}$ Bryan D. Quaife and M. Nicholas J. Moore. A boundary-integral framework to simulate viscous erosion of a porous medium. Journal of Computational Physics, 375:1-21, 2018.

${ }^{57}$ Karen Alim, Shima Parsa, David A. Weitz, and Michael P. Brenner. Local Pore Size Correlations Determine Flow Distributions in Porous Media. Physical Review Letters, 119(14):1-5, 2017.

${ }^{58}$ Sujit S. Datta, H. Chiang, T. S. Ramakrishnan, and David A. Weitz. Spatial fluctuations of fluid velocities in flow through a three-dimensional porous medium. Phys. Rev. Lett., 111:064501, Aug 2013.

${ }^{59}$ B. R. Corrochano, J. R. Melrose, A. C. Bentley, P. J. Fryer, and S. Bakalis. A new methodology to estimate the steady-state permeability of roast and ground coffee in packed beds. Journal of Food Engineering, 150:106-116, 2015.

${ }^{60}$ Borja Roman-Corrochano. Advancing the Engineering Understanding of Coffee Extraction. PhD thesis, University of Birmingham, 2015.

${ }^{61}$ Verena Bernadette Hargarten, Michael Kuhn, and Heiko Briesen. Swelling properties of roasted coffee particles. Journal of the Science of Food and Agriculture, 100(10):3960-3970, 2020.

${ }^{62}$ Matthew J. Maille, Kyle Sala, David M. Scott, and Hannah Zukswert. Critical examination of particle swelling during wetting of ground coffee. Journal of Food Engineering, 295(November 2020):110420, 2020.

${ }^{63}$ C. D'Onofrio, R. van Loon, S. Rolland, R. Johnston, L. North, S. Brown, R. Phillips, and J. Sienz. Three-dimensional computational model of a blood oxygenator reconstructed from micro-CT scans. Medical Engineering and Physics, 47:190-197, 2017.

${ }^{64}$ H. C. Burridge, G. Wu, T. Reynolds, D. U. Shah, R. Johnston, O. A. Scherman, M. H. Ramage, and P. F. Linden. The transport of liquids in softwood: timber as a model porous medium. Scientific Reports, 9(1):1-14, 2019. 